\title{
LEXML
}

LexML Brasil

Parte 3 - LexML XML Schema

Versão 1.0 (RC1)

Brasília - Dezembro de 2008 


\section{LexML Brasil}

\section{Parte 3 - LexML XML Schema}

Versão 1.0 (Dezembro/2008)

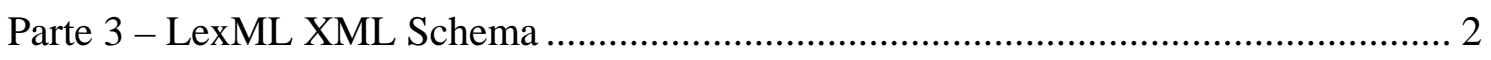

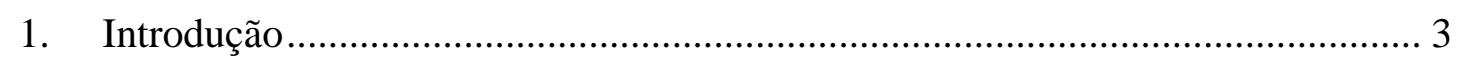

2. Esquemas Rígido e Flexível para Texto Articulado........................................... 3

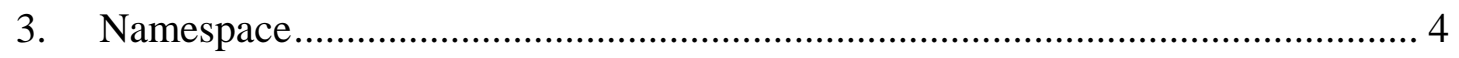

4. Nome e Conteúdo de Elementos e Atributos ...................................................... 4

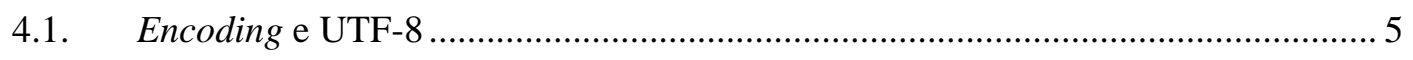

4.2. Cuidados na Codificação de Alguns Caracteres..................................................... 5

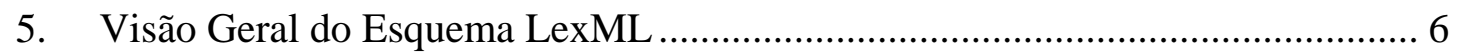

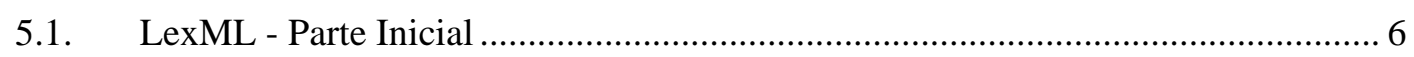

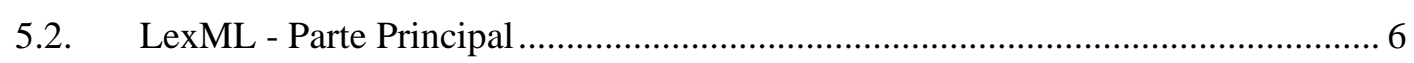

5.3. Identificadores de Agrupamento de Artigo .......................................................... 7

5.4. Identificadores de Artigos e Dispositivos de Artigo ……………........................... 8

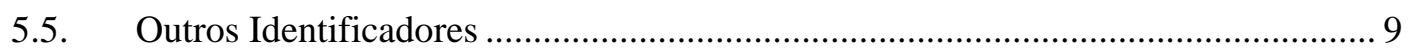

6. Estrutura de Acórdão em LexML .................................................................... 9

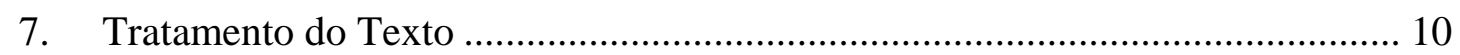

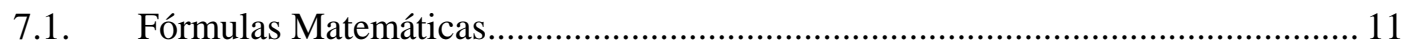

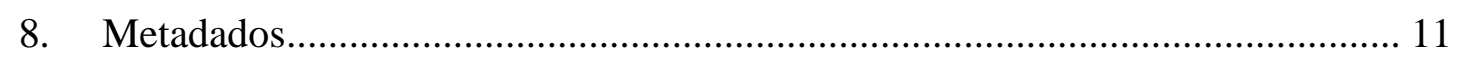

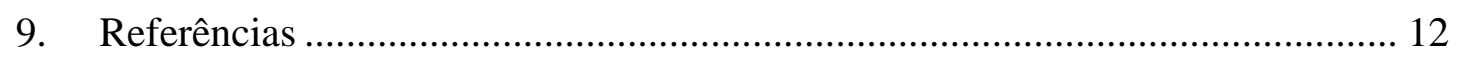

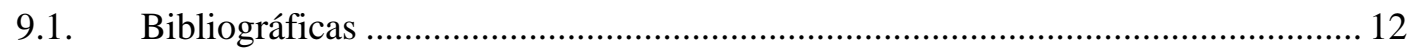

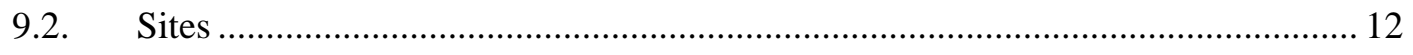

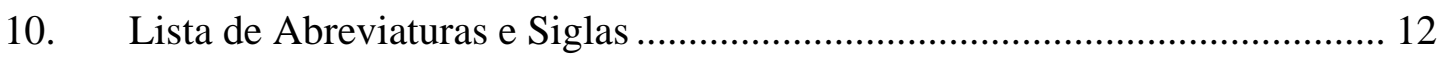

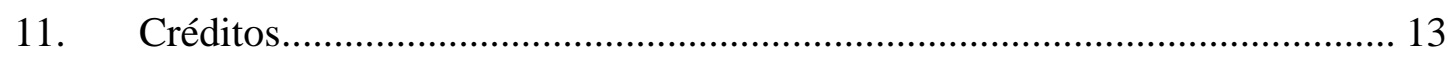

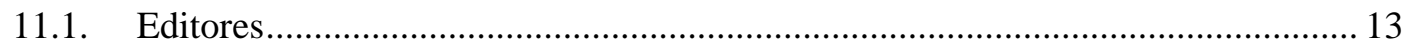

11.2. Grupo de Trabalho LexML (em Dezembro de 2008)............................................. 13

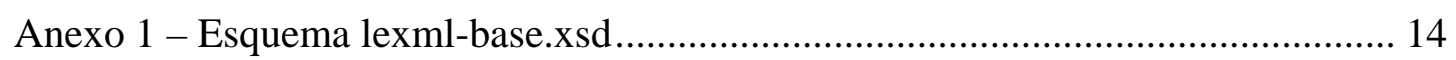

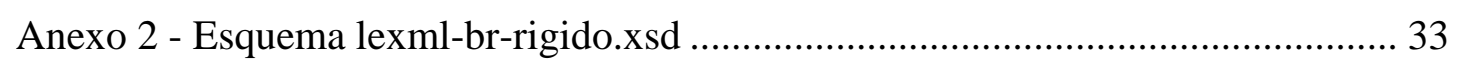

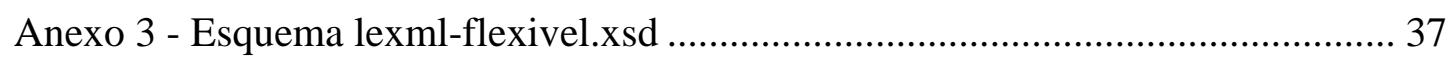




\section{Introdução}

Este documento apresenta princípios e conceitos utilizados na definição do esquema XML para a estruturação dos textos de normas, julgados e projetos de normas.

O esquema do Projeto LexML Brasil é baseado nos esquemas dos Projetos NIR (Norme in Rete) e AKOMA NTOSO, ambos editados pelo Prof. Dr. Fabio Vitali, da Universidade de Bolonha. Serão enfatizados nas próximas seções aspectos referentes ao ordenamento jurídico brasileiro e à técnica legislativa federal. Em algumas seções, haverá adaptação e/ou tradução de trechos do documento de referência do projeto AKOMA NTOSO.

Na época da implantação do projeto NIR, a linguagem XML Schema ainda estava em processo de definição. Atualmente, o esquema do Projeto NIR está disponível tanto na notação DTD (Document Type Definition) quanto em XML Schema. O Projeto LexML Brasil utiliza apenas a linguagem XML Schema para modelagem dos documentos textuais por essa linguagem possuir mais recursos de validação, quando comparado com a DTD.

A leitura deste documento requer o conhecimento dos padrões $X M L$ (W3C 2006) e de XML Schema (W3C 2004).

\section{Esquemas Rígido e Flexível para Texto Articulado}

Devido à falta de padronização da técnica legislativa em relação às três esferas e à existência de normas federais com articulação incompatível com a Lei Complementar $n^{0}$ 95/1998 (principalmente normas anteriores a 1998), foram criados dois esquemas, a exemplo da estratégia do Projeto NIR, conforme abaixo:

- Esquema Rígido, de acordo com a Lei Complementar no 95/1998 - Normas e projetos de normas que seguem as regras de articulação definidas pela Lei Complementar $n^{\circ}$ 95/1998.

- Esquema Flexível - Permite mais combinações na articulação dos dispositivos. Aplica-se às normas e proposições legislativas que não seguem a Lei Complementar $n^{\circ}$ 95/1998 além de servir como um denominador comum entre os esquemas rígidos definidos para cada técnica legislativa.

As definições comuns fazem parte do Esquema Base que é incluído nos Esquemas Rígido e Flexível.

Todo documento válido no Esquema Rígido é também válido no Esquema Flexível. No entanto, o inverso não é verdadeiro.

Estados e municípios que não utilizam as regras de estruturação conforme a Lei Complementar no 95/1998, devem definir seu próprio Esquema Rígido. Por exemplo, será necessário definir um Esquema Rígido de acordo com a técnica legislativa do Estado de São Paulo, definida pela Lei Complementar Estadual $n^{0}$ 863/1999. As 
instâncias de documentos XML válidas no Esquema Rígido da LCP-SP 863/1999 também serão válidas no Esquema Flexível do LexML.

Normas federais anteriores a 1998 que não consigam ser validadas no Esquema Rígido LCP 95/1998 devem utilizar o Esquema Flexível.

\section{Namespace}

As instâncias de documentos LexML são completamente qualificadas, isto é, utiliza-se o namespace "http://www.lexml.gov.br/1.0” como namespace default para todos os elementos.

Apesar de alguns elementos utilizarem o mesmo nome de elementos HTML, para simplificar, decidiu-se incorporar alguns elementos do namespace HTML ao namespace LexML. Como resultado, é possível especificar o namespace LexML como namespace default minimizando o uso de prefixos no documento da instância, ao mesmo tempo em que se mantém a qualificação completa dos elementos.

O LexML utiliza três atributos que devem ser codificados com o prefixo do namespace explícito, conforme a seguir:

o "xml:lang" e "xml:base" do namespace

http : //WWW.w3 . org/XML/1998/namespace, e

o “xlink:href” do namespace http://WWW.w3.org/1999/xlink.

O atributo xml:lang deve ser utilizado no elemento inline <span> para delimitar expressões escritas em outras línguas, tais como: latim (“la”), inglês (“en”), espanhol (“es”) e alemão (“de”).

O atributo xml:base deve ser utilizado no elemento <Alteracao> ou $<$ RemissaoMultipla $>$ para identificar a URN da norma que está sendo alterada ou referenciada. Todos os atributos xlink:href que fazem parte dos elementos contidos nos elementos <Alteracao> e <Remissaomultipla> são relativos à URN indicada no atributo xml:base.

\section{Nome e Conteúdo de Elementos e Atributos}

Uma das principais características de um documento XML é a sua capacidade de se auto-descrever, isto é, cada segmento do conteúdo textual é delimitado por marcações (tags) que possuem nomes significativos.

Todos os elementos do LexML (exceto os “importados” de outros vocabulários - ex. HTML) utilizam a notação UpperCamelCase. Os atributos utilizam a notação lowerCamelCase, isto é, iniciam com letra minúscula e cada nova palavra é iniciada por letra maiúscula.

Ao se definir uma nova linguagem na notação XML é comum surgirem dúvidas quanto à escolha entre o construtor elemento e atributo em uma determinada situação. Utilizou-se a seguinte diretriz no LexML: o conteúdo textual dos elementos deve ser 
suficiente para reproduzir o texto constante do documento oficial (em papel ou arquivo digitalmente assinado) na ordem em que ele se apresenta; os atributos codificam informações de controle do conteúdo texto, tais como identificadores, referências, indicação da língua etc. No caso de acórdãos, onde, em alguns casos, há repetição do cabeçalho, decidiu-se codificar estas informações em um elemento <CabecalhoAcordao> separado dos outros componentes textuais do acórdão (relatório, voto etc) com o objetivo de minimizar a redundância de informações.

Não são utilizados diacríticos nem a letra "ç” nos nomes de elementos, atributos ou no conteúdo de atributos “id”. Por serem construtores voltados principalmente para o processamento técnico do documento, faz parte da prática da informática omitir estes sinais que nem sempre estão disponíveis nos teclados.

\subsection{Encoding e UTF-8}

As instâncias de documentos do LexML utilizam o encoding “UTF-8”. Esta é a codificação default para o XML e foi escolhida porque oferece o armazenamento em um único byte para os caracteres mais comuns e permite a utilização dos caracteres definidos no padrão Unicode. A codificação ISO-8859-1 (muito difundida no Brasil) sempre utiliza um único byte por caractere, e, por isso, é bem mais limitada que a UTF8. Por exemplo, a codificação ISO-8859-1 não possui as aspas angulares: “ (abre aspas) e " (fecha aspas). Seria necessário utilizar entidades ou referências numéricas para codificá-las em um documento XML caso fosse utilizada a codificação ISO-8859-1.

Outra vantagem da UTF-8 é a possibilidade de armazenamento de documentos textuais de outros países que utilizam conjuntos de caracteres não latinos.

\subsection{Cuidados na Codificação de Alguns Caracteres}

É muito comum encontrar na Internet textos de normas codificados com caracteres errados. Um dos casos mais comuns é a codificação errada do Símbolo Ordinal “ o ” (código 186), utilizado na identificação de normas e dispositivos, como por exemplo: "Lei $n^{\circ} 8.112$..." ou " $\S 2^{\circ}$ do art. 60". Este símbolo algumas vezes é codificado como “ o ” (letra “o” minúscula sobrescrita) ou como “ o ” (Símbolo Grau código 176).

A palavra abreviada “números” deve ser codificada como “nº" (letra “n”; símbolo de ordinal (código 186); letra "s” sem marcação de estilo sobrescrito ou sublinhado).

De acordo com o texto da Lei Complementar $n^{0}$ 95/1998, os incisos são codificados em números romanos e separados do texto por hífen “-” (código 45). Neste caso não devem ser utilizados os símbolos ene “ - ” (código 150) ou eme “— ” (código 151).

A codificação errada de caracteres pode gerar vários problemas, como, por exemplo, erro na pronúncia por parte dos softwares de síntese de voz utilizados por 
deficientes visuais ou pessoas com baixa visão ou, ainda, erro na utilização de software de recuperação da informação ou de verificação ortográfica.

\section{Visão Geral do Esquema LexML}

A Parte Inicial apresenta construtores (grupos, tipos etc.) que são utilizados pela Parte Principal. Como o XML Schema do LexML foi derivado do XML Schema do Projeto AKOMA NTOSO, foram preservados a forma de organização e alguns comentários originais. Alguns construtores da parte inicial estão definidos com nome em inglês. No entanto, esses nomes são utilizados apenas internamente no esquema e não são visíveis nas instâncias de documentos LexML.

\subsection{LexML - Parte Inicial}

A parte inicial do esquema relaciona alguns grupos de elementos (<xsd: groups >) e atributos (<xsd:attributeGroup >) utilizados na definição dos modelos de conteúdos e tipos em todo o documento. Na seqüência, são definidos tipos simples (<xsd : simpleType>), basicamente para enumeração de valores string, e tipos complexos (<xsd: complexType $>$ ). Esses tipos complexos correspondem a Design Patterns de modelos de conteúdo referenciados na definição dos elementos conforme (Vitali et. al, 2005) e (Vitali, 2007).

Hierarchy - elementos de estrutura hierárquica (ex: <Livro>, <Parte>, etc.).

Block - seqüência de elementos bloco (ex: $<p>)$.

Inline - trata elementos de conteúdo misto (ex: conteúdo de $<\mathrm{p}>$ ).

Marker - elemento de conteúdo vazio (ex: <NotaReferenciada/>).

O Design Pattern Container não possui um tipo complexo associado, a exemplo dos outros. Cada elemento dessa categoria organiza os elementos na ordem desejada, utilizando os elementos construtores da linguagem XML Schema.

\subsection{LexML - Parte Principal}

Todos os documentos LexML compartilham o mesmo elemento raiz <LexML>, cujo conteúdo inicial é obrigatoriamente o elemento <Metadado> seguido do elemento que identifica o tipo de documento. A utilização de um único elemento raiz segue o Design Pattern "Universal Root”.

O LexML prevê inicialmente os seguintes tipos de documentos:

Norma - Constituição, Emendas, Leis, Decretos etc.

ProjetoNorma - Proposições legislativas.

Jurisprudencia - Súmulas e Acórdãos.

DocumentoGenerico - Para outros tipos de documentos.

Anexo - Anexos dos documentos acima. 
A Norma e o Projeto de Norma utilizam o tipo Hierarchicalstructure; os Anexos podem ser definidos utilizando tanto uma estrutura hierárquica quanto uma estrutura genérica, dependendo do caso específico. A Jurisprudência utiliza a estrutura genérica para cada um dos seus componentes textuais (Relatório, Voto, etc).

$\mathrm{Na}$ seção seguinte do esquema, são definidos containers referenciados na definição dos tipos de documento. Por exemplo, é possível encontrar nesta seção os elementos ParteInicial, Articulacao e ParteFinal, referenciados na definição do elemento Norma.

Os elementos que definem a hierarquia de um documento articulado, de acordo com a Lei Complementar $n^{0}$ 95/1998, são apresentados no arquivo "lexml-br-rigido.xsd”. Inicialmente são apresentados os elementos que agrupam artigos (Parte, Livro, Titulo, Capitulo, Secao e Subsecao), em seguida a unidade básica de articulação (Artigo), e, por fim, os dispositivos de artigo (Caput, Inciso, Alinea, Item, Paragrafo). Os elementos correspondentes, no esquema flexível, são apresentados no arquivo “lexml-flexivel.xsd”. Os esquemas rígido e flexível fazem inclusão do esquema base, localizado no arquivo "lexml-base.xsd".

Na seqüência, são apresentados os elementos genéricos correspondentes a cada um dos cinco patterns de modelo de conteúdo: Agrupamento (Container), AgrupamentoHierarquico (Hierarchy), Bloco (Block), EmLinha (Inline), Marca (Marker). Ao utilizar estes elementos genéricos é necessário especificar o atributo "nome" significativo.

Em seguida, o esquema apresenta os elementos “importados” do vocabulário HTML que tratam da codificação do texto corrido, de listas, de tabelas e de outros elementos de formatação tais como negrito, itálico, sublinhado, subscrito e sobrescrito. Trata-se de uma simplificação do vocabulário HTML.

Por fim, são apresentados os elementos de metadados que tratam de vários aspectos, tais como identificação, contexto, indexação, ciclo de vida e informações sobre a publicação.

\subsection{Identificadores de Agrupamento de Artigo}

A definição de identificadores para os dispositivos tem por objetivo identificar partes de um documento. No esquema LexML, os identificadores são permitidos em todos os elementos, e, em alguns, devido a sua importância, são atributos obrigatórios. Eles podem ser utilizados, por exemplo, nas remissões textuais de um dispositivo ou na indicação de qual dispositivo está sendo alterado.

Os identificadores de elementos que agrupam artigos são criados a partir da concatenação do identificador de cada nível hierárquico desde o nível mais alto até o nível que se está identificando. Por exemplo, id="tit1_cap2-1_sec3" é o identificador da "Seção III do Capítulo II-A do Título I". Note que os algarismos romanos e as letras são convertidos em números arábicos. A letra do seqüencial de inclusão também é convertida para o número arábico. No caso do elemento <Parte>, 
normalmente identificado por um nome (Parte Geral, Parte Especial), utiliza-se um seqüencial a partir do número 1.

A Tabela 2 apresenta os identificadores de agrupamento de artigo previstos na Lei Complementar $\mathrm{n}^{0}$ 95/1998 bem como o elemento genérico <AgrupamentoHierarquico> que poderá ser utilizado nos casos não previstos. Por exemplo, o novo Código Civil utiliza o elemento "Subtítulo" não previsto na Lei Complementar $n^{\circ}$ 95/1998.

Em algumas situações, será necessário utilizar o Esquema Flexível que permite mais combinações dos elementos que agrupam artigos.

Tabela 2. Identificadores para agrupamento de artigos.

\begin{tabular}{|c|c|c|c|}
\hline Elemento & id & Exemplo & id do exemplo \\
\hline$<$ Parte $>$ & prtN & $\begin{array}{l}\text { Parte Geral do Código } \\
\text { Civil }\end{array}$ & prt1 \\
\hline$<$ Livro $>$ & $\operatorname{livN}$ & Livro I & liv1 \\
\hline$<$ Titulo> & titN & Título II & tit2 \\
\hline <Capitulo> & capN & $\begin{array}{l}\text { Título IX, Capítulo IV- } \\
\text { A }\end{array}$ & tit9_cap4-1 \\
\hline$<$ Secao $>$ & $\sec \mathrm{N}$ & Seção III do Capítulo I & cap1_sec3 \\
\hline <Subsecao> & subN & $\begin{array}{l}\text { Parte Geral do Código } \\
\text { Civil, Livro I, Título } \\
\text { VI, Capítulo I, Seção } \\
\text { II, Subseção I }\end{array}$ & prt2_liv1_tit6_cap1_sec2_sub1 \\
\hline <AgrupamentoHierarquico> & aghN & $\begin{array}{l}\text { Subtítulo I do Título II } \\
\text { do Livro II da Parte } \\
\text { Especial do Código } \\
\text { Civil }\end{array}$ & prt2_liv2_tit2_agh1 \\
\hline
\end{tabular}

\subsection{Identificadores de Artigos e Dispositivos de Artigo}

A lógica de formação dos identificadores de artigos e seus dispositivos é similar à dos elementos que agrupam artigos, atentando-se ao fato da presença do elemento <Caput> que não possui o seqüencial numérico por ele ser único e obrigatório.

A Tabela 3 apresenta os identificadores de artigo e seus dispositivos previstos na Lei Complementar $\mathrm{n}^{\circ}$ 95/1998 bem como o elemento genérico <DispositivoGenerico> que poderá ser utilizado nos casos de dispositivos não previstos ou em desacordo com os da lei complementar. Em algumas situações será necessário utilizar o Esquema Flexível que permite mais combinações entre os dispositivos de artigos. A tabela mostra também exemplos em que as letras das alíneas são convertidas em números arábicos. 
Tabela 3. Identificadores de Artigo e Dispositivos de Artigo.

\begin{tabular}{|c|c|c|c|}
\hline Elemento & Id & Exemplo(s) & id do exemplo \\
\hline$<$ Artigo $>$ & $\operatorname{artN}$ & Art. $5^{\circ}$ & art5 \\
\hline$<$ Caput> & cpt & caput do art. $5^{\circ}$ & art5_cpt \\
\hline$<$ Paragrafo $>$ & parN & Art. 8-A, parágrafo único & art8-1_par1 \\
\hline <Inciso $>$ & incN & $\begin{array}{l}\text { inciso III do art } 8^{\circ} \\
\text { inciso II do } \S 2^{\circ} \text { do art } 20\end{array}$ & $\begin{array}{l}\text { art8_cpt_inc3 } \\
\text { art20_par2_inc2 }\end{array}$ \\
\hline$<$ Alinea $>$ & aliN & $\begin{array}{l}\text { Alínea “a” do inciso IX do } \\
\text { parágrafo único do art. } 3^{\circ} \text {-A- } \\
\text { A. }\end{array}$ & art3-1-1_par1_inc9_ali1 \\
\hline$<$ Item $>$ & iteN & $\begin{array}{l}\text { item 1, da alínea “b”, do } \\
\text { inciso I, do art. } 39\end{array}$ & art39_cpt_inc1_ali2_ite1 \\
\hline$<$ DispositivoGenerico $>$ & dpgN & alínea "b” do $\S 3^{\circ}$ do art. $5^{\circ}$ & art5_par3_dpg2 \\
\hline
\end{tabular}

Na seção de definição dos tipos simples do esquema, foram definidos patterns com expressões regulares para validação dos identificadores.

\subsection{Outros Identificadores}

Os identificadores servem de "gancho" para a localização de determinado partição do documento. No caso de normas jurídicas, foram definidos alguns identificadores para rápida localização de outros segmentos de texto, tais como: epigrafe, ementa, preambulo, localdata e fecho.

Os identificadores de dispositivos alvo de uma alteração (normalmente o novo texto destes dispositivos aparece entre aspas) são precedidos pelo prefixo CONT_aspN_, onde CONT (contexto) representa o id do dispositivo da alteração na norma publicada e $\mathrm{N}$ representa o seqüencial das aspas referente à alteração. Por exemplo, o identificador art14_cpt_asp1_art4_cpt_inc3 identifica o elemento com a alteração do Inciso III do art. $4^{\circ}$ da norma identificada no elemento <Alteracao>. Este elemento está localizado na primeira seqüência de aspas do caput do art 14 da norma alteradora.

\section{Estrutura de Acórdão em LexML}

O inteiro teor de um Acórdão é formado por seis elementos conforme a seguir:

- <CabecalhoAcordao> - contém dados estruturados comuns como epígrafe, data de julgamento, órgão julgador e partes.

- <EmentaTexto> - texto livre da ementa.

- <AcordaoTexto> - texto livre com o acórdão.

- $<$ RelatorioTexto > - texto livre com o relatório.

- $\quad$ VotoTexto $>$ - texto livre com o voto.

- <DebateTexto> - texto livre opcional com debates. 
- <ExtratoAtaTexto> - texto livre com o Extrato da Ata.

A estrutura para texto livre do LexML permite a codificação de texto com sofisticadas características, tais como definição de links persistentes para normas (de qualquer esfera) e julgados.

As notas de rodapé são tratadas em cada componentes de texto do acórdão utilizando o elemento $<$ div $>$ e links bidirecionais. Dessa forma é possível isolar este elemento ao mesmo tempo em que é permitida a navegação entre a nota e o texto principal.

O elemento "class" pode ser codificado em cada parágrafo para tratar as questões de apresentação em uma folha de estilo CSS.

\section{Tratamento do Texto}

Com o intuito de facilitar o desenvolvimento de ferramentas para redação de documentos no formato LexML, definimos a utilização de um subconjunto de elementos HTML para a estruturação da parte textual do documento.

Os elementos importados desempenham o mesmo papel daquele definido para o HTML. Acrescenta-se a esta regra apenas uma exceção: o elemento <div >, que no HTML desempenha o papel de bloco genérico, no LexML desempenha o papel de container genérico, pois o elemento $<\mathrm{p}>$ já desempenha o papel de bloco genérico.

Os elementos $\langle$ div $\rangle,\langle p\rangle$ e $<$ span $>$ são considerados elementos genéricos de container, bloco e inline, respectivamente, sendo equivalentes a <Agrupador $>$, $<$ Bloco $>$ e $<$ EmLinha $>$.

Dentro de um elemento $<\mathrm{p}>$ é possível utilizar o elemento inline $<$ Remissao $>$ para criar referências href para outros documentos do LexML.

As referências poderão ser absolutas ou relativas. Este último caso ocorre quando o elemento <Remissao> está codificado internamente ao elemento $<$ Alteracao $>$ ou <RemissaoMultipla>: no elemento remissão será codificada a referência relativa para o id do dispositivo apontado (href="art1_par1"), enquanto que no elemento <Alteracao $>$ haverá a referência absoluta para a norma ( xml: base="urn: lex: br ; sao . paulo: lei : 2004-02-11;123:").

Para otimizar a marcação de referências, nos casos em que muitos dispositivos de uma mesma norma são referenciados, é possível utilizar o elemento $<$ Remissaomultipla $>$ que conterá mais de um elemento <Remissao $>$. O primeiro utiliza o atributo xml:base, e, o segundo, atributo xlink:href com referências relativas.

Todos os elementos do esquema LexML (inclusive os "importados") podem ser marcados com atributos class e style, permitindo a associação de estilos CSS com definições precisas de apresentação. 


\subsection{Fórmulas Matemáticas}

Para incluir fórmulas matemáticas deve-se utilizar o elemento inline <Formula> que inclui o elemento <math> do vocubulário MathML (namespace http://www.w3.org/1998/Math/MathML). A fórmula poderá ser codificada em um arquivo externo ou no próprio documento LexML.

\section{Metadados}

O elemento Metadado contém informações sobre o documento para atender a diversas necessidades tais como recuperação da informação, preservação e gerência do ciclo de vida do documento.

Como regra, todo conteúdo feito por um editor (e não pelo autor) deve fazer parte desta seção. Vice versa, todo conteúdo do autor não deve ser codificado na seção de metadados.

A Seção < Metadados> está estruturada em oito subseções, conforme a seguir:

o Identificação - contém elementos de metadados que identificam unicamente o documento corrente.

o Contexto - identifica a posição do arquivo corrente na hierarquia FRBR. A posição é indicada pelo atributo ' $\mathrm{id}=$ "self"' do elemento FRBRItem.

o CicloDeVida - registra os eventos relacionados ao arquivo corrente. No caso de textos multivigentes, deve-se registrar o histórico de eventos do período relativo à vigência do documento.

o EventosGerados - registra os eventos de alteração que afetam outros documentos. Por exemplo, a alteração e revogação de dispositivos de outras normas.

o Notas - registra notas do editor ou do responsável pelo markup. As notas podem ser visíveis, nos casos em que registre informações sobre a versão corrente, ou invisíveis, nos casos em que faz-se um registro técnico sobre o markup.

o Recursos - lista alguns recursos auxiliares que podem referenciados em outras pontos da seção de metadados.

o MetadadosProprietario - ponto de extensão para registro de metadados não considerados no atual esquema.

Para o detalhamento dos campos da Seção Identificação, deve-se consultar o documento URN (LexML - Parte 2) e para o entendimento da hierarquia FRBR no contexto do Projeto LexML, deve-se consultar o documento Modelo de Referência (LexML - Parte 1). 


\section{Referências}

\subsection{Bibliográficas}

VITALI, F; Di IORIO, A.; GUBELLINI, D.; Design Patterns for Descriptives Document Substructures. In Extreme Markup Language 2005. Montreal, Québec. 2005. Acessado em 03 de Janeiro de 2007. Disponível em: [www.mulberrytech.com/Extreme/Proceedings/html/2005/Vitali01/EML2005Vitali01.html]

VITALI, F. Akoma Ntoso 1.0 Release Notes. (versão 23/11/2006). 2006. Acessado em 3 de fevereiro de 2007. Disponível em: [ http://www.akomantoso.org/ AKOMA_NTOSO/downloads-1/Release_Notes_20112006.pdf ]

W3C-2004. XML Schema Part 0: Primer Second Edition. W3C recommendation. Disponível em : http://www.w3.org/TR/xmlschema-0/. Acessado em 01 de dezembro de 2008.

W3C-2006. Extensible Markup Language (XML) 1.0: W3C recommendation. Disponível em: http://www.w3.org/TR/2006/REC-xml-20060816/. Acesso em 01 de dezembro de 2008.

\subsection{Sites}

http://www.nir.it/

Projeto Norme in Rete (Itália)

http://www.akomantoso.org/

Projeto Akoma Ntoso (Nações Unidas/África)

http://www.xmlpatterns.com/

Design Patterns para XML

http://www.w3c.org

Recomendações do W3C

\section{Lista de Abreviaturas e Siglas}

CSS - Cascade Styling Sheets

DTD - Document Type Definition

FRBR - Functional Requirements for Bibliographical Records

HTML - Hypertext Markup Language

NIR - Norme in Rete

XML - Extensible Markup Language

W3C - World Wide Web Consortium 


\section{Créditos}

\subsection{Editores}

João Alberto de Oliveira Lima (Senado Federal / Prodasen)

Fernando Ciciliati (Senado Federal / Interlegis)

\subsection{Grupo de Trabalho LexML (em Dezembro de 2008)}

Alfredo Luiz Campos Júnior (Câmara dos Deputados / CENIN)

Carlos Corrêa Gonçalves (Tribunal Superior Eleitoral)

Cláudio Morale (Senado Federal / Interlegis)

Cláudio Mori (Tribunal Superior Eleitoral)

Claudson dos Santos Melo (Tribunal Superior do Trabalho)

Dalva Luca (Ministério da Justiça)

Fernando Teixeira (Câmara dos Deputados / CENIN)

Flávia Lacerda Oliveira de Macedo (Tribunal de Contas da União)

Flávio Henrique Rocha e Silva (Supremo Tribunal Federal)

Flávio Heringer (Senado Federal)

Manuel de Medeiros Dantas (Advocacia Geral da União)

Jean Rodrigo Ferri (Senado Federal / Interlegis)

João Alberto de Oliveira Lima (Senado Federal / Prodasen)

João Batista de Holanda Neto (Senado Federal / Prodasen)

João R. Kramer Santana (Tribunal de Contas da União)

Jonatas Rodrigues (Procuradoria Geral da República)

Luís Henrique Raja G. Mitchell (Tribunal de Contas da União)

Paulo André Mattos de Carvalho (Tribunal de Contas da União)

Paulo de Tarso Penna da Costa (Superior Tribunal de Justiça)

Paulo Martins Inocêncio (Conselho da Justiça Federal)

Ricardo Bravo (Tribunal de Contas da União)

Sérgio Falcão (Câmara dos Deputados / CENIN)

Virgínia Azevedo (Supremo Tribunal Federal) 


\title{
Anexo 1 - Esquema lexml-base.xsd
}

\author{
$<$ ?xml version="1.0" encoding="UTF-8"?> \\ <xsd:schema targetNamespace="http://www.lexml.gov.br/1.0" \\ xmlns="http://www.lexml.gov.br/1.0" \\ xmlns:xlink="http://www.w3.org/1999/xlink" \\ xmlns:mathml="http://www.w3.org/1998/Math/MathML" \\ elementFormDefault="qualified" \\ xmlns:xsd="http://www.w3.org/2001/XMLSchema">
}

<xsd:import namespace="http://www.w3.org/1999/xlink" schemaLocation="Xlink.xsd" />

<xsd:import namespace="http://www.w3.org/XML/1998/namespace" schemaLocation="http://www.w3.org/2001/xml.xsd"/>

<xsd:import namespace="http://www.w3.org/1998/Math/MathML" schemaLocation="mathml2/mathml2.xsd"/>

$<$ xsd:annotation>

$<$ xsd:documentation>

O esquema do projeto LexML Brasil é uma adaptação dos schemas

Akoma Ntoso (1.0) (http://www.akomantoso.org/)

Norme in Rete (2.0) (http://www.nir.it)

para a técnica legisativa brasileira. Esses esquemas foram

originalmente criados por Fabio Vitali ( Universidade of Bolonha )

com a supervisão (Direito) de Monica Palmirani ( Universidade de Bolonha ).

Adaptado por João Alberto de Oliveira Lima (Senado Federal - joaolima@senado.gov.br)

e revisado por Fernando Ciciliati (Interlegis - ciciliati@interlegis.gov.br)

O presente esquema usa XML design patterns de forma sistemática, tal como descrito em http://www.xmlpatterns.com/

Alguns deles são mencionados explicitamente, quando o seu uso

não é suficientemente claro ou natural. Outros são usados sem nenhuma

referência.

Versão 1.0 - dezembro/08 - exclusão dos elementos Texto e TextoSimples

Versão 0.7 - agosto/08 - ajuste no patterns de ids de dispositivos para trocar o complemento alfa para num - inclusão do elemento inline Formula com conteúdo MathML.

Versão 0.6 - abril/08 - alteração nos ids das subdivisões de artigo retirando o 'alt' e incluindo o prefixo do dispositivo pai.

- inclusão do elemento Pena após o Texto dos dispostivos de artigo.

- alteração de nome da tag "Justificativa" para "Justificacao

- inclusão do elemento EmConformidadeCom

- inclusão de elemento Apelido no nível ObraIndividual

e ObraIndividualComplementar

\section{$<$ xsd:documentation>}

$</$ xsd:annotation $>$

$<$ xsd:annotation>

$<$ xsd:documentation $>$

Principais Grupos de Elementos

- LexML (Hierarchy, Inline, Marker)

- HTML (Container, Block, Inline, Marker)

- LexML + HTML + generic elements

$</$ xsd:documentation $>$

$</$ xsd:annotation $>$

<xsd:group name="LXhier">

$<$ xsd:choice $>$

$<$ xsd:element ref="Parte"/>

$<$ xsd:element ref="Livro"/>

$<$ xsd:element ref="Titulo"/>

<xsd:element ref="Capitulo"/> 


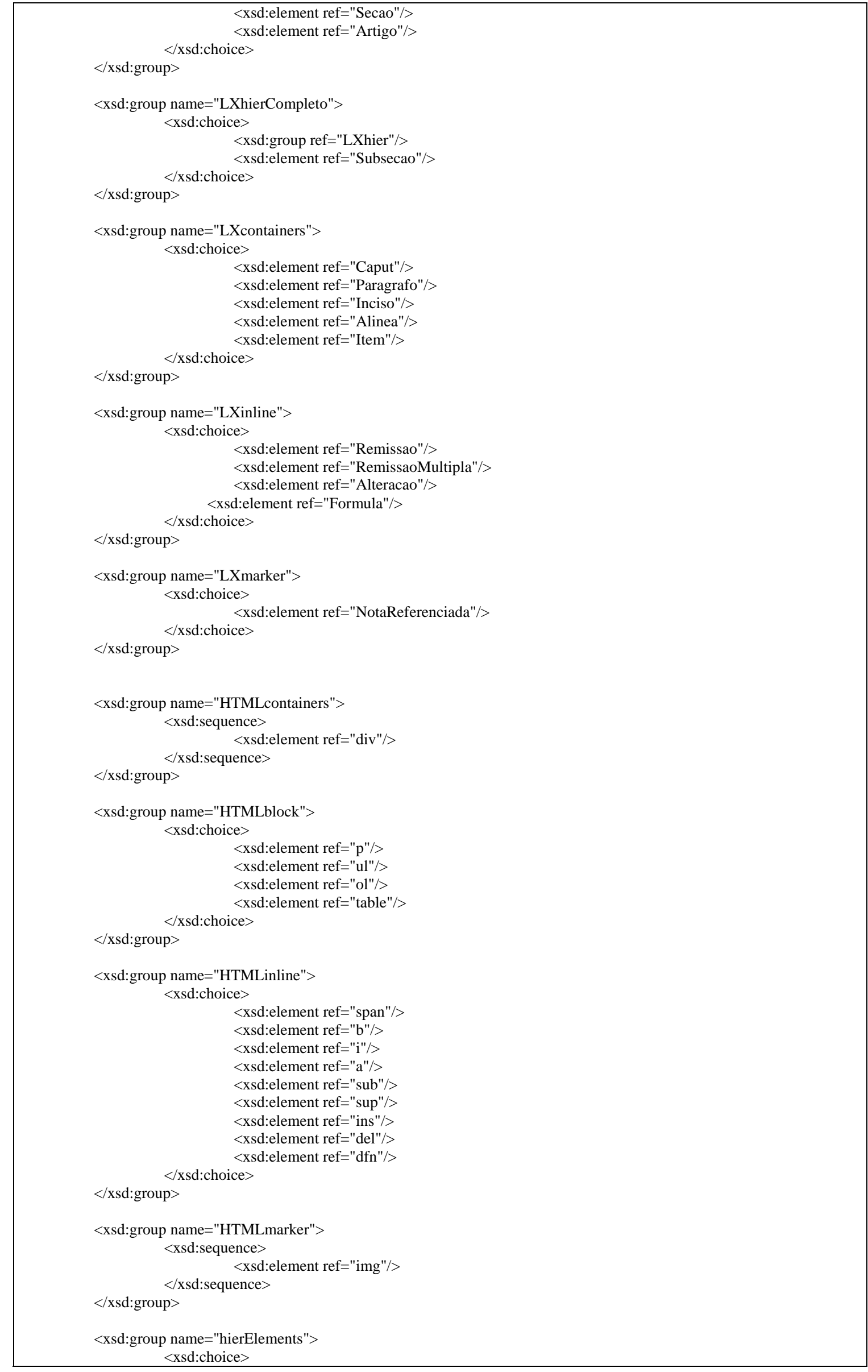




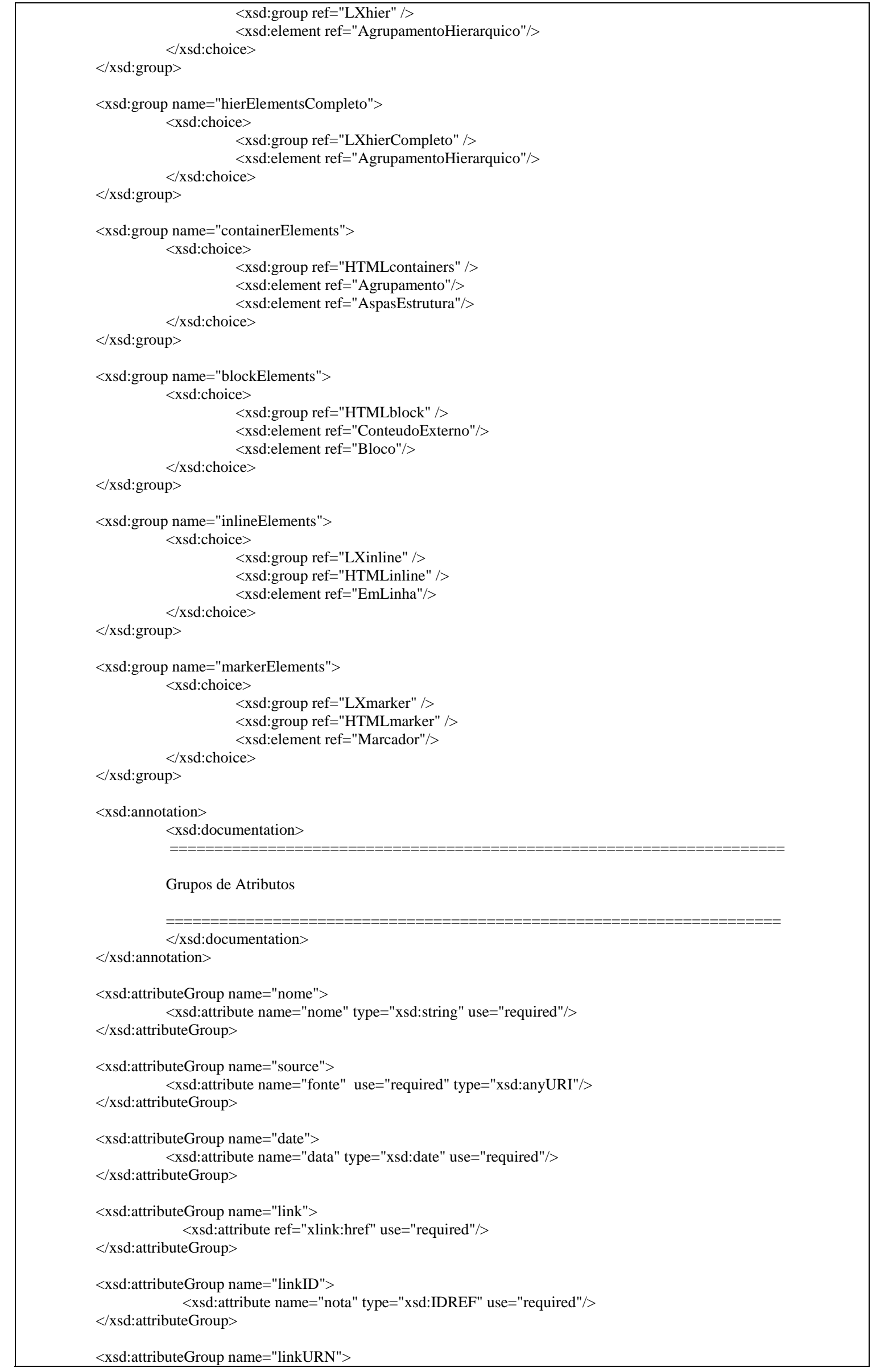


$</$ xsd:attributeGroup $>$

$<$ xsd:attribute name="URN" type="xsd:anyURI" use="required"/>

$<$ xsd:attribute name="showAs" type="xsd:string"/>

<xsd:attributeGroup name="linkopt">

$<$ xsd:attribute ref="xlink:href"/>

$</$ xsd:attributeGroup $>$

$<$ xsd:attributeGroup name="optvalue" $>$

$<$ xsd:attribute name="value" type="xsd:string"/>

$</$ xsd:attributeGroup $>$

$<$ xsd:attributeGroup name="period">

$<$ xsd:attribute name="eventoInicial" type="xsd:anyURI"/>

$<$ xsd:attribute name="eventoFinal" type="xsd:anyURI"/>

$</$ xsd:attributeGroup $>$

<xsd:attributeGroup name="enactment" $>$

$<$ xsd:attributeGroup ref="period"/>

$<$ xsd:attribute name="situacao" type="TipoSituacao"/>

$</$ xsd:attributeGroup $>$

$<$ xsd:attributeGroup name="cellattrs" >

$<$ xsd:attribute name="rowspan" type="xsd:integer" default="1" />

$<$ xsd:attribute name="colspan" type="xsd:integer" default="1" />

$</$ xsd:attributeGroup $>$

$<$ xsd:attributeGroup name="HTMLattrs" $>$

$<$ xsd:attribute name="class" type="xsd:string" $>>$

$<$ xsd:attribute name="style" type="xsd:string"/>

$<$ xsd:attribute name="title" type="xsd:string"/>

$</$ xsd:attributeGroup $>$

$<$ xsd:attribute ref="xml:lang" default="pt-BR"/>

$<$ xsd:attributeGroup name="HTMLname">

$<$ xsd:attribute name="name" type="xsd:string"/>

$</$ xsd:attributeGroup $>$

$<$ xsd:attributeGroup name="idreq">

$<$ xsd:attribute name="id" use="required" type="xsd:ID"/>

$<$ xsd:attribute name="seqOrdem" type="xsd:int"/>

$</$ xsd:attributeGroup $>$

$<$ xsd:attributeGroup name="idreqArt">

$<$ xsd:attribute name="id" use="required" type="idArtigo"/>

$<$ xsd:attribute name="seqOrdem" type="xsd:int"/>

$</$ xsd:attributeGroup $>$

$<$ xsd:attributeGroup name="idopt" $>$

$<$ xsd:attribute name="id" type="xsd:ID"/>

$</$ xsd:attributeGroup $>$

$<$ xsd:attributeGroup name="corereq" $>$

$<$ xsd:attributeGroup ref="HTMLattrs"/>

$<$ xsd:attributeGroup ref="enactment"/>

$<$ xsd:attributeGroup ref="idreq"/>

$</$ xsd:attributeGroup $>$

$<$ xsd:attributeGroup name="coreopt" >

$<$ xsd:attributeGroup ref="HTMLattrs"/>

$<$ xsd:attributeGroup ref="enactment"/>

$<$ xsd:attributeGroup ref="idopt"/>

$</$ xsd:attributeGroup $>$

<xsd:attributeGroup name="corereqArt" >

$<$ xsd:attributeGroup ref="HTMLattrs"/>

$<$ xsd:attributeGroup ref="enactment"/>

$<$ xsd:attributeGroup ref="idreqArt"/>

$</$ xsd:attributeGroup $>$

$<$ xsd:annotation $>$

$<$ xsd:documentation $>$

Tipos Simples 


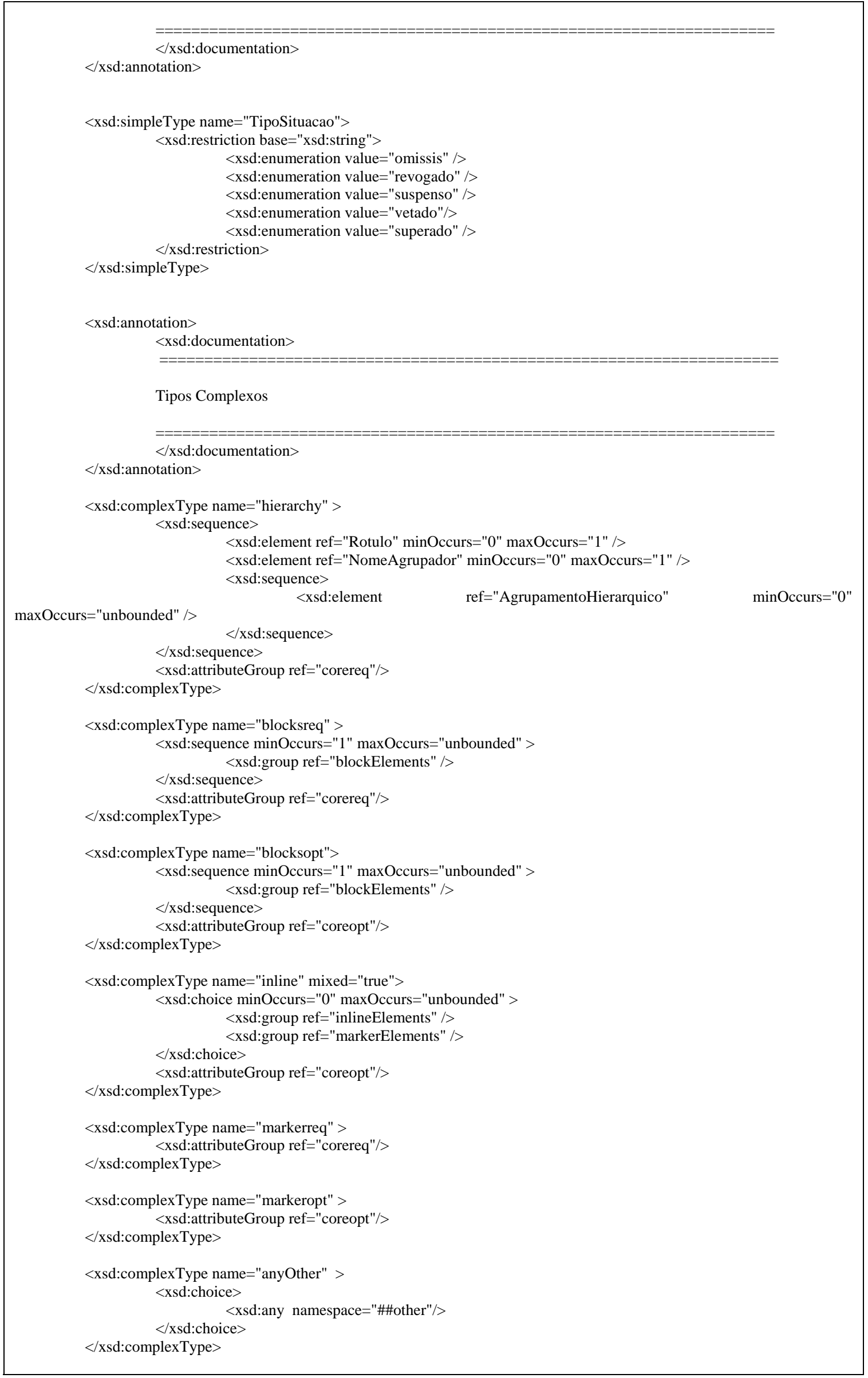


$<$ xsd:complexType name="participaType" >

$<$ xsd:sequence $>$

<xsd:element ref="Rotulo" minOccurs="1" maxOccurs="1" />

$<$ xsd:element ref="Tratamento" minOccurs="0" maxOccurs="1" />

$<$ xsd:element ref="NomeAgente" minOccurs="1" maxOccurs="1" />

$</$ xsd:sequence $>$

$</$ xsd:complexType $>$

$<$ xsd:complexType name="textoType" $>$

$<$ xsd:sequence $>$

$</$ xsd:sequence $>$

$<$ xsd:element ref="p" minOccurs="0" maxOccurs="unbounded" />

$<$ xsd:attributeGroup ref="corereq"/>

$</$ xsd:complexType $>$

$<$ xsd:complexType name="textoSimplesType">

$<$ xsd:sequence $>$

$<$ xsd:element ref="p" minOccurs="0" maxOccurs="1" />

$<$ xsd:sequence $>$

$<$ xsd:attributeGroup ref="corereq"/>

$<$ xsd:complexType $>$

<xsd:complexType name="textoSimplesOptType">

$<$ xsd:sequence $>$

$<$ xsd:element ref="p" minOccurs="0" maxOccurs="1" />

$</$ xsd:sequence $>$

$</$ xsd:complexType $>$

$<$ xsd:attributeGroup ref="coreopt"/>

$<$ xsd:annotation $>$

$<$ xsd:documentation $>$

Elemento Raiz

Comentário Um elemento genérico raiz contendo todos os tipos de documentos

Pattern: Universal Root

Referência: http://www.xmlpatterns.com/UniversalRootMain.shtml

$</$ xsd:documentation $>$

$</$ xsd:annotation $>$

$<$ xsd:complexType name="DocumentTypes" >

$<$ xsd:sequence $>$

$<$ xsd:element ref="Metadado" / >

$<$ xsd:choice $>$

$<$ xsd:element ref="Norma" />

$<$ xsd:element ref="ProjetoNorma" />

$<$ xsd:element ref="Jurisprudencia" />

$<$ xsd:element ref="DocumentoGenerico" />

$<$ xsd:choice $>$

$</$ xsd:sequence $>$

$<$ xsd:element ref="Anexo" />

$</$ xsd:complexType $>$

$<$ xsd:element name="LexML" type="DocumentTypes" $>>$

$<$ xsd:annotation $>$

$<$ xsd:documentation $>$

Tipos de Documento

Comentário: Todos os tipos de documentos compartinham dois modelos de conteúdo (Hierárquico, Aberto)

Pattern: Consistent Element Set

Referência: http://www.xmlpatterns.com/ConsistentElementSetMain.shtml

$</$ xsd:documentation>

$</$ xsd:annotation $>$

$<$ xsd:complexType name="HierarchicalStructure" >

$<$ xsd:sequence $>$

$<$ xsd:element ref="ParteInicial" minOccurs="0" maxOccurs="1" />

$<$ xsd:element ref="Articulacao" />

$<$ xsd:element ref="ParteFinal" minOccurs="0" maxOccurs="1" /> 


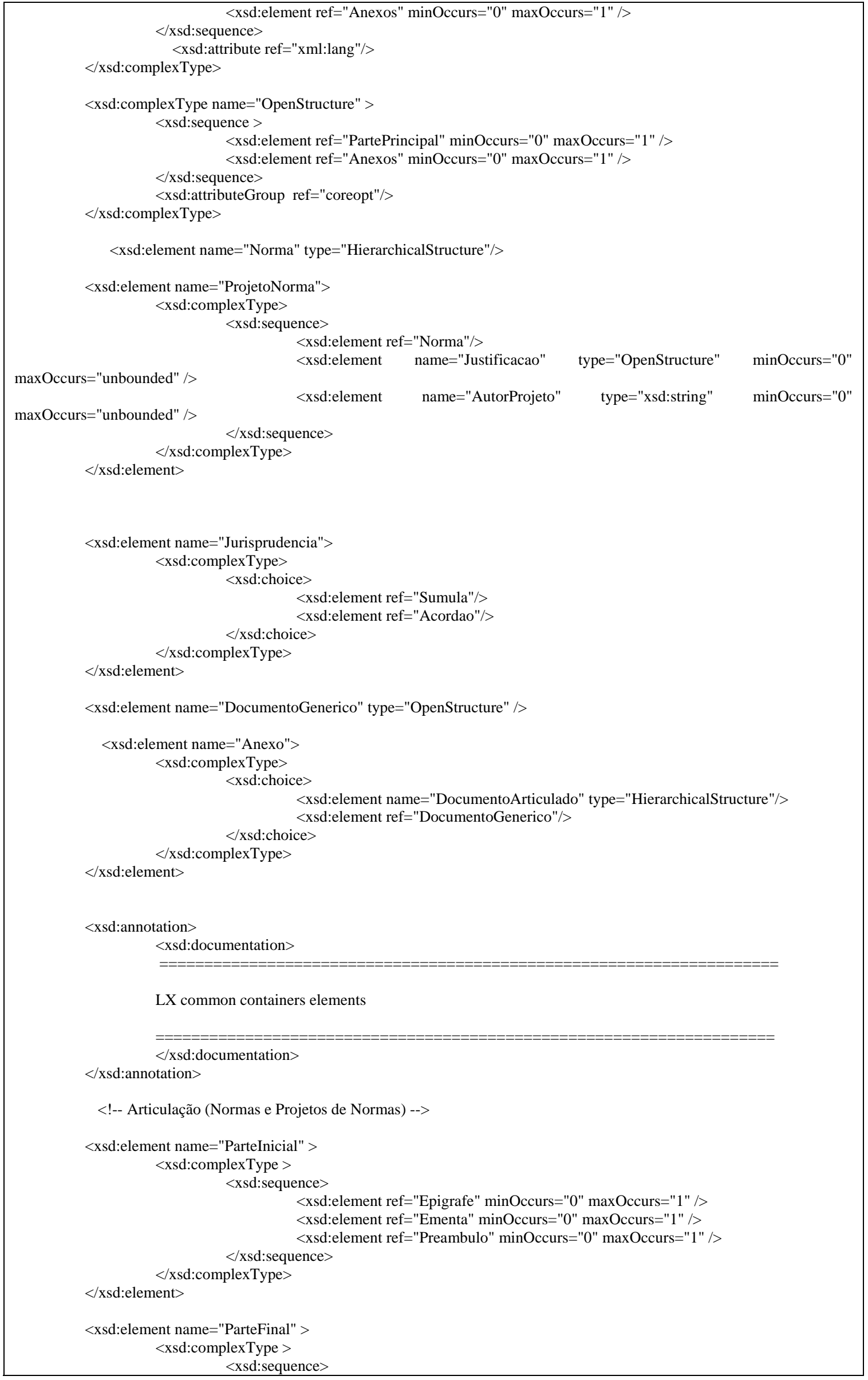




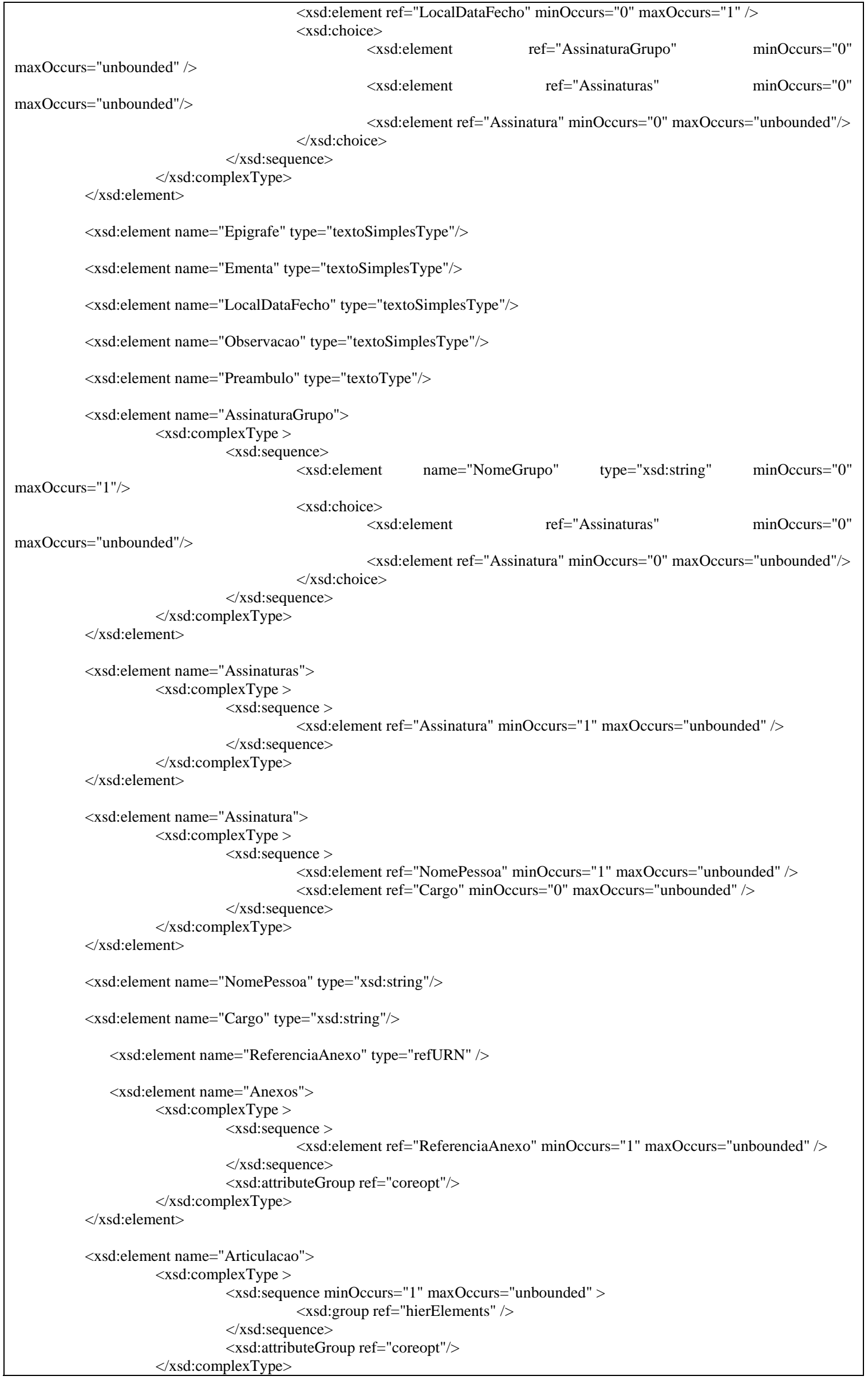




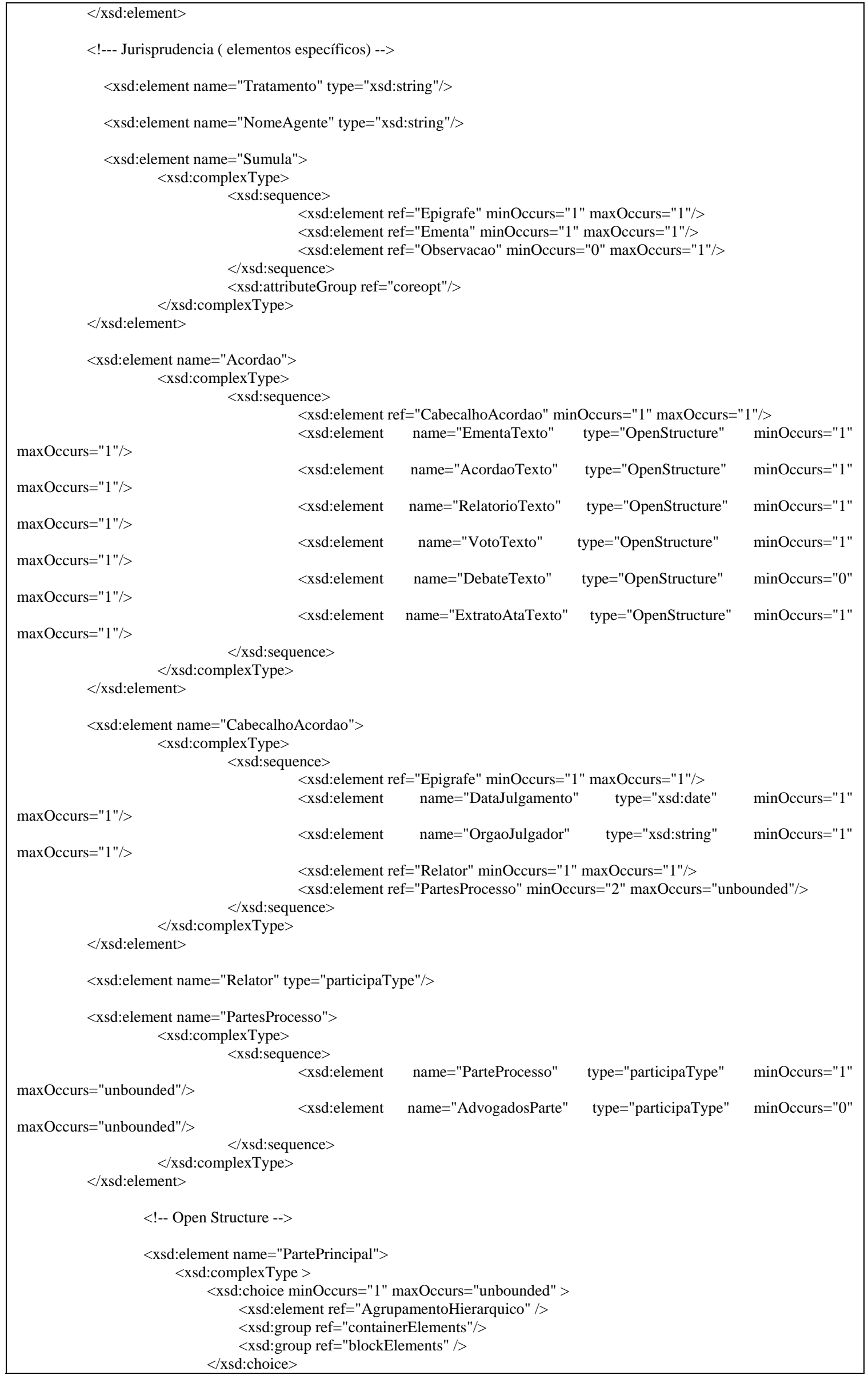




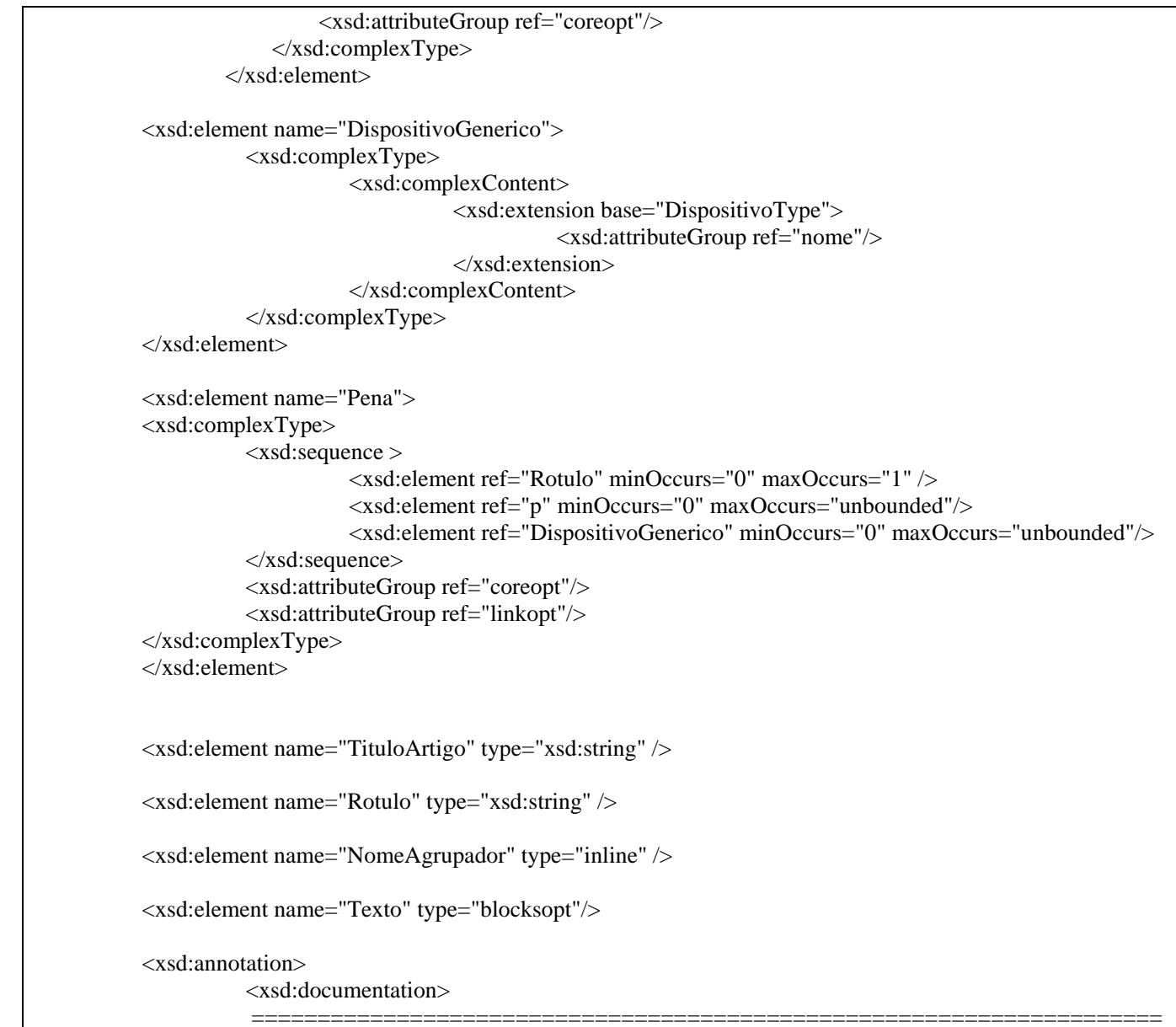

\section{LX elementos inline}

$</$ xsd:documentation $>$

$</$ xsd:annotation $>$

$<$ xsd:element name="Remissao">

$<$ xsd:complexType mixed="true">

$<$ xsd:complexContent>

<xsd:extension base="inline">
$\quad<$ xsd:attributeGroup ref="link"/>
</xsd:extension>

$</$ xsd:complexContent $>$

$</$ xsd:element $>$

$<$ xsd:complexType $>$

<xsd:element name="RemissaoMultipla">

$<$ xsd:complexType mixed="true">

$<$ xsd:complexContent>

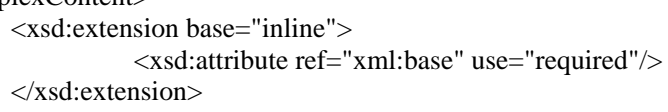

$</$ xsd:complexContent $>$

$</$ xsd:element $>$

$<$ xsd:complexType $>$

<xsd:element name="NotaReferenciada">

$<$ xsd:complexType>

$<$ xsd:complexContent $>$

<xsd:extension base="markeropt">

$</$ xsd:extension $>$

<xsd:attributeGroup ref="linkID"/>

$</$ xsd:complexContent $>$

$</$ xsd:complexType $>$

$</$ xsd:element $>$ 
$<$ xsd:element name="Alteracao" $>$

<xsd:complexType mixed="true">

$<$ xsd:complexContent $>$

<xsd:extension base="inline">

$<$ xsd:sequence $>$

$<$ xsd:choice $>$

maxOccurs="unbounded" />

$<$ xsd:element ref="AspasTexto" minOccurs="1"

maxOccurs="unbounded" />

<xsd:element ref="AspasEstrutura" minOccurs="1"

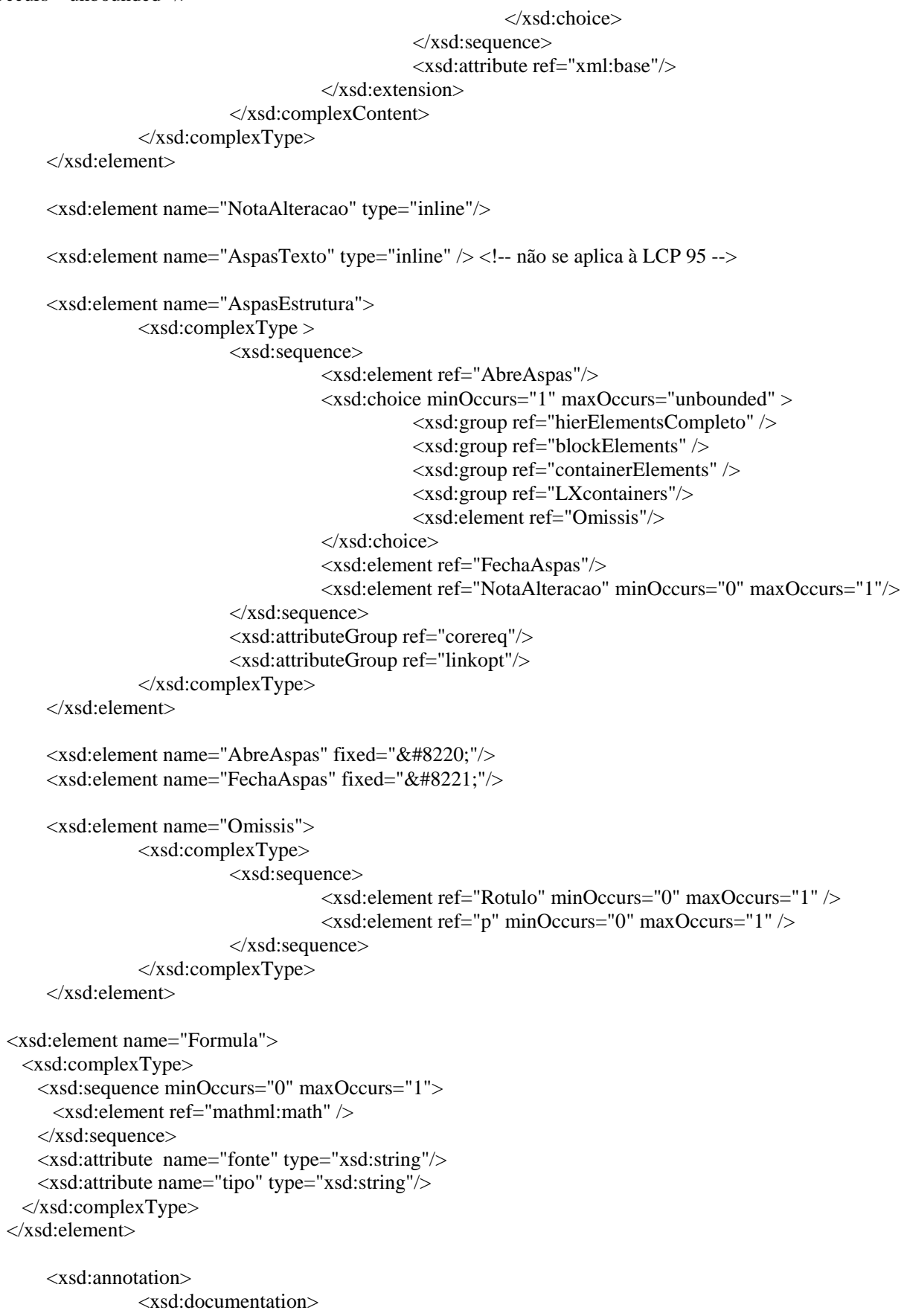




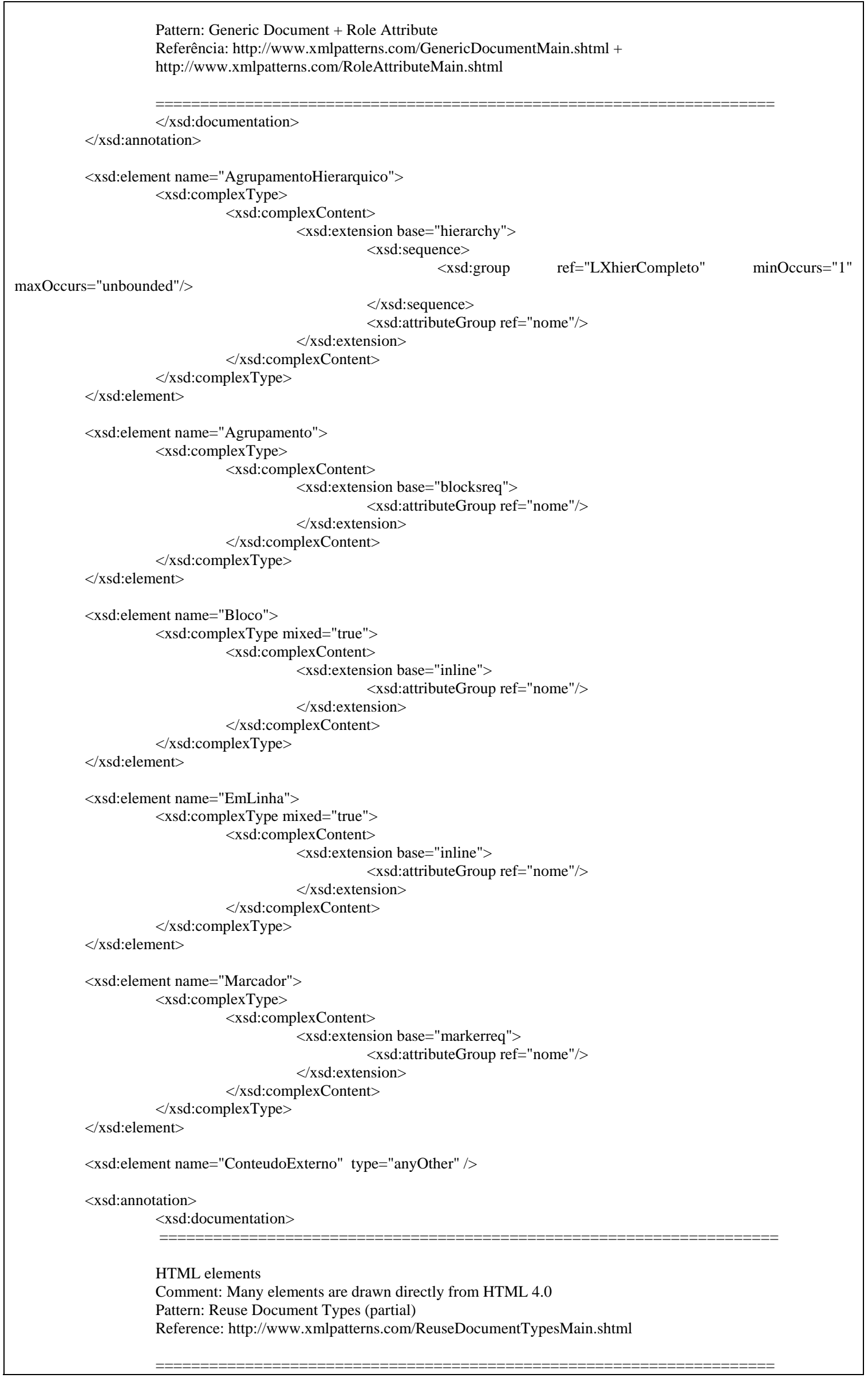


$</$ xsd:annotation $>$

<xsd:element name="div" type="blocksreq" />

$<$ xsd:element name="p" type="inline" />

<xsd:element name="li">

$<$ xsd:complexType mixed="true" $>$

$<$ xsd:complexContent>

$<$ xsd:extension base="inline" $>$

<xsd:choice minOccurs="0" maxOccurs="unbounded" >

$<$ xsd:element ref="ul" />

$<$ xsd:element ref="ol" />

$</$ xsd:choice $>$

$<$ xsd:element ref="p"/>

$</$ xsd:extension $>$

$</$ xsd:complexContent $>$

$</$ xsd:element $>$

$<$ xsd:complexType $>$

<xsd:element name="span" type="inline"/>

$<$ xsd:element name="b" type="inline" />

$<$ xsd:element name="i" type="inline" />

<xsd:element name="sub" type="inline" />

<xsd:element name="sup" type="inline" />

$<$ xsd:element name="ins" type="inline" />

$<$ xsd:element name="del" type="inline" $/>$

<xsd:element name="dfn" type="inline" />

<xsd:element name="a">

$<$ xsd:complexType mixed="true">

$<$ xsd:complexContent>

$<$ xsd:extension base="inline" $>$

<xsd:attributeGroup ref="link"/>

$<$ xsd:attributeGroup ref="HTMLname"/>

$</$ xsd:extension $>$

xsd:attribute name="target" type="xsd:string"/>

$</$ xsd:complexContent $>$

$</$ xsd:element $>$

xsd:complexType $>$

<xsd:element name="img">

<xsd:complexType>

$<$ xsd:complexContent>

<xsd:extension base="markeropt">

<xsd:attribute name="src" type="xsd:anyURI" use="required"/>

$<$ xsd:attribute name="alt" type="xsd:string"/>

$<$ xsd:attribute name="width" type="xsd:integer"/>

$<$ xsd:attribute name="height" type="xsd:integer"/> $<$ xsd:extension $>$

$<$ xsd:complexContent $>$

$</$ xsd:complexType $>$

$</$ xsd:element $>$

<xsd:element name="ul">

$<$ xsd:complexType >

$<$ xsd:sequence $>$

$</$ xsd:sequence $>$

$<$ xsd:element ref="li" minOccurs="1" maxOccurs="unbounded" />

$</$ xsd:element $>$

$</$ xsd:complexType $>$

<xsd:element name="ol">

$<$ xsd:complexType >

$<$ xsd:sequence $>$

$<$ xsd:element ref="li" minOccurs="1" maxOccurs="unbounded" /> 
$</$ xsd:element $>$

$</$ xsd:sequence $>$

$</ x s d:$ complexType $>$

$<$ xsd:element name="table" $>$

$<$ xsd:complexType $>$

$<$ xsd:sequence $>$

$<$ xsd:element ref="tr" minOccurs="1" maxOccurs="unbounded" />

$</$ xsd:sequence $>$

$<$ xsd:attributeGroup ref="HTMLattrs"/>

$<$ xsd:attribute name="width" type="xsd:integer"/>

$<$ xsd:attribute name="border" type="xsd:integer"/>

$<$ xsd:attribute name="cellspacing" type="xsd:integer"/>

$<$ xsd:attribute name="cellpadding" type="xsd:integer"/>

$<$ xsd:attributeGroup ref="idreq"/>

$</$ xsd:element $>$

$</$ xsd:complexType $>$

$<$ xsd:element name="tr" >

$<$ xsd:complexType >

$<$ xsd:choice minOccurs="1" maxOccurs="unbounded" >

$<$ xsd:element ref="th" $/>$

$<$ xsd:choice $>$

$<$ xsd:element ref="td" $>>$

$<$ xsd:attributeGroup ref="HTMLattrs"/>

$</$ xsd:element $>$

$<$ xsd:complexType $>$

$<$ xsd:element name="th" $>$

$<$ xsd:complexType mixed="true">

$<$ xsd:complexContent>

$<$ xsd:extension base="inline" $>$

$<$ xsd:attributeGroup ref="cellattrs"/> $</$ xsd:extension $>$

$</$ xsd:complexContent $>$

$</$ xsd:complexType $>$

$</$ xsd:element $>$

$<$ xsd:element name="td">

$<$ xsd:complexType mixed="true">

$<$ xsd:complexContent>

$<$ xsd:extension base="inline" $>$

$<$ xsd:attributeGroup ref="cellattrs"/> $</$ xsd:extension $>$

$<$ /xsd:complexContent $>$

$</$ xsd:complexType $>$

$</$ xsd:element $>$

$<$ xsd:annotation $>$

$<$ xsd:documentation $>$

Metadados

$</$ xsd:documentation $>$

$</$ xsd:annotation $>$

<!-- Algumas definições de tipos preliminares -->

<xsd:simpleType name="MetadadoLiteralTexto">

$<$ xsd:restriction base="xsd:string">

$<$ xsd:minLength value $=" 1 " />$

$</$ xsd:restriction $>$

$</$ xsd:simpleType $>$

$<$ xsd:complexType name="MetadadoLiteralTextoURN">

$<$ xsd:simpleContent>

$<$ xsd:extension base="MetadadoLiteralTexto">

$<$ xsd:attribute name="URN" type="xsd:anyURI"/>

$</$ xsd:extension $>$

$</$ xsd:simpleContent $>$

$</$ xsd:complexType $>$

<xsd:simpleType name="MetadadoData"> 


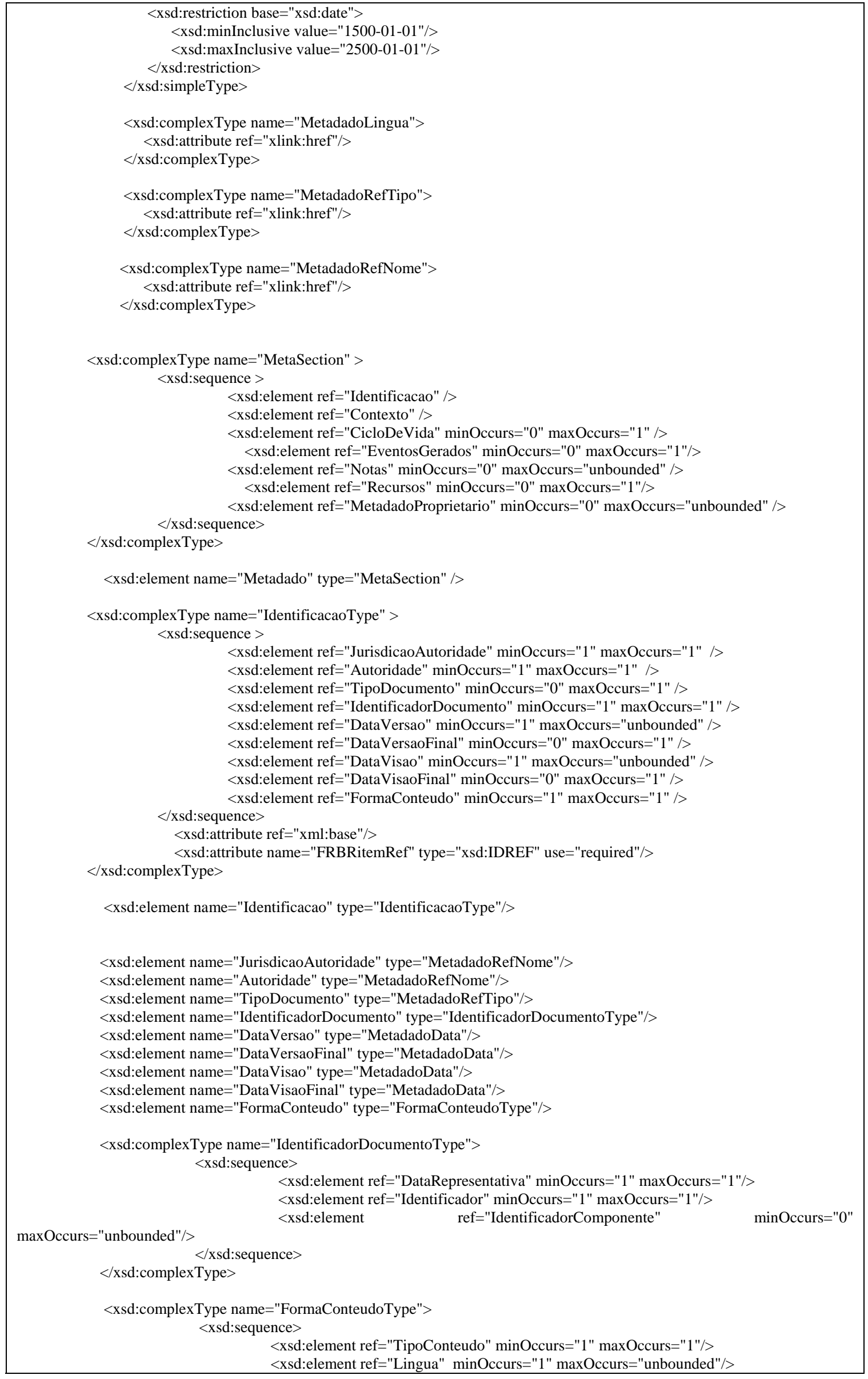


$</$ xsd:complexTypd:sequence $>$

</xsd:

$<$ xsd:element name="DataRepresentativa" type="MetadadoData"/>

$<$ xsd:element name="Identificador" type="MetadadoLiteralTexto"/>

$<$ xsd:element name="IdentificadorComponente" type="MetadadoLiteralTexto"/>

<xsd:element name="TipoConteudo" type="MetadadoRefTipo"/>

$<$ xsd:element name="Lingua" type="MetadadoLingua"/>

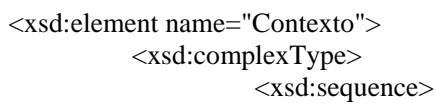




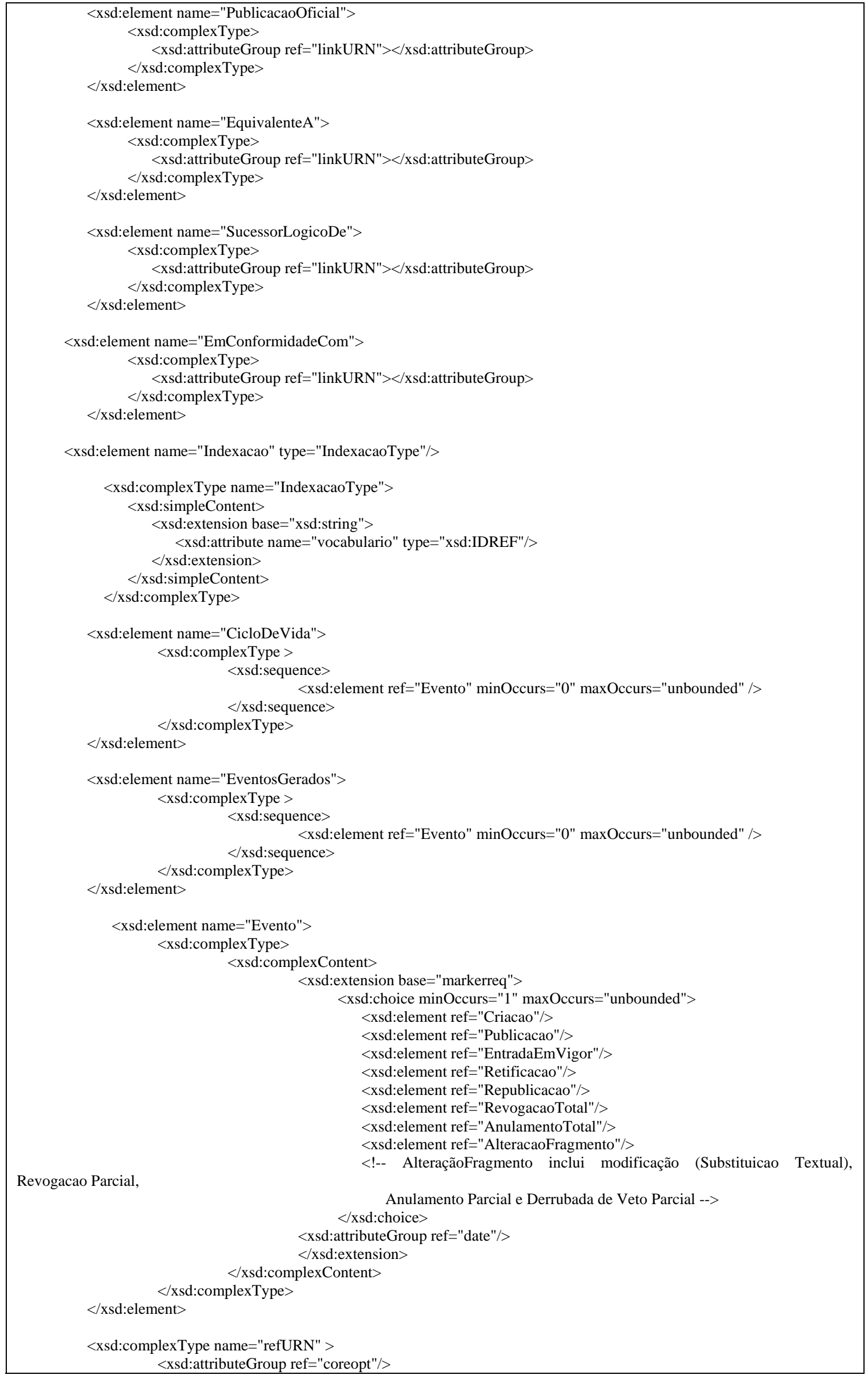


$</$ xsd:complexType $>$

<xsd:attribute name="AlvoURN" type="xsd:anyURI"/>

$<$ xsd:attribute name="FonteURN" type="xsd:anyURI"/>

$<$ xsd:complexType name="refURNSimples" >

$<$ xsd:attributeGroup ref="coreopt"/>

$<$ /xsd:complexType $>$

$<$ xsd:attribute name="URN" type="xsd:anyURI"/>

<xsd:element name="Publicacao" type="refURN" |>

$<$ xsd:element name="EntradaEmVigor" type="refURN"/>

$<$ xsd:element name="Republicacao" type="refURN" $>$

$<$ xsd:element name="Retificacao" type="refURN" />

$<$ xsd:element name="ProjetoNormaOrigem" type="refURN" / >

$<$ xsd:element name="JulgadoOrigemAnulacao" type="refURN" / >

<xsd:element name="NormaFonte" type="refURN"/>

<xsd:element name="MensagemVetoAplicado" type="refURN" />

$<$ xsd:element name="RevogacaoTotal" type="refURN"/>

$<$ xsd:element name="AnulamentoTotal" type="refURN"/>

$<$ xsd:element name="Criacao">

$<$ xsd:complexType $>$

$<$ xsd:sequence $>$

$<$ xsd:element ref="ProjetoNormaOrigem" minOccurs="0" maxOccurs="unbounded"/>

$<$ xsd:element ref="JulgadoOrigemAnulacao" minOccurs="0" maxOccurs="unbounded"/>

<xsd:element ref="MensagemVetoAplicado" minOccurs="0" maxOccurs="unbounded"/>

$</$ xsd:sequence $>$

$</$ xsd:complexType $>$

$</$ xsd:element $>$

<xsd:complexType name="refURNAlvo" >

$<$ xsd:attributeGroup ref="coreopt"/>

$<$ xsd:attribute name="FonteURN" type="xsd:anyURI"/>

$<$ xsd:attribute name="AlvoURN"/>

$<$ xsd:attribute name="AlvoLocal" type="xsd:IDREFS"/>

$</$ xsd:complexType $>$

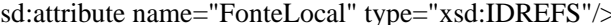

<xsd:complexType name="URNtype" >

$<$ xsd:attributeGroup ref="coreopt"/>

$<$ xsd:attribute name="URN" type="xsd:anyURI"/>

$</$ xsd:complexType $>$

$<$ xsd:element name="AlteracaoFragmento">

$<$ xsd:complexType $>$

$<$ xsd:sequence $>$

$<$ xsd:element ref="ModificacaoTextual" minOccurs="0" maxOccurs="unbounded"/>

$<$ xsd:element ref="RevogacaoParcial" minOccurs="0" maxOccurs="unbounded"/>

$<$ xsd:element ref="AnulamentoParcial" minOccurs="0" maxOccurs="unbounded"/>

$<$ xsd:element ref="DerrubadaVetoParcial" minOccurs="0" maxOccurs="unbounded"/>

$</$ xsd:sequence $>$

$</$ xsd:complexType $>$

$</$ xsd:element $>$

$<$ xsd:element name="ModificacaoTextual" type="refURNAlvo"/>

$<$ xsd:element name="RevogacaoParcial" type="refURNAlvo"/>

$<$ xsd:element name="AnulamentoParcial" type="refURNAlvo"/>

$<$ xsd:element name="DerrubadaVetoParcial" type="refURNAlvo"/>

$<$ xsd:element name="Notas" $>$

$<$ xsd:complexType >

<xsd:sequence minOccurs="1" maxOccurs="unbounded" >

$<$ xsd:element ref="Nota" / >

$</$ xsd:sequence $>$

$</$ xsd:complexType $>$ 
$</$ xsd:element $>$

$<$ xsd:element name="Nota" $>$

$<$ xsd:complexType $>$

$<$ xsd:complexContent $>$

$<$ xsd:extension base="textoType">

$<$ xsd:attribute name="exporta" type="xsd:boolean"/>

$<$ xsd:attribute name="dataInclusao" type="xsd:date"/>

$<$ xsd:attribute name="autor" type="xsd:IDREF"/>

$<$ xsd:extension $>$

$</$ xsd:complexContent $>$

$</$ xsd:complexType $>$

$</$ xsd:element $>$

$<$ xsd:element name="Recursos" $>$

$<$ xsd:complexType >

$<$ xsd:sequence minOccurs="1" maxOccurs="unbounded" >

$<$ xsd:element ref="Recurso" $>>$

$</$ xsd:complexType $>$

$</$ xsd:element $>$

$</$ xsd:sequence $>$

$<$ xsd:element name="Recurso" type="markerreq" / >

$<$ xsd:element name="MetadadoProprietario">

$<$ xsd:complexType $>$

$<$ xsd:complexContent $>$

$<$ xsd:extension base="xsd:anyType">

$<$ xsd:attributeGroup ref="source"/>

$</$ xsd:extension $>$

$</$ xsd:complexContent $>$

$</$ xsd:element $>$

$<$ xsd:complexType $>$

$<$ xsd:annotation>

$<$ xsd:documentation $>$

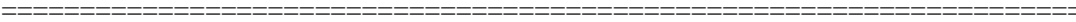

End of Schema

$</$ xsd:documentation>

$</$ xsd:annotation $>$ 


\section{Anexo 2 - Esquema lexml-br-rigido.xsd}

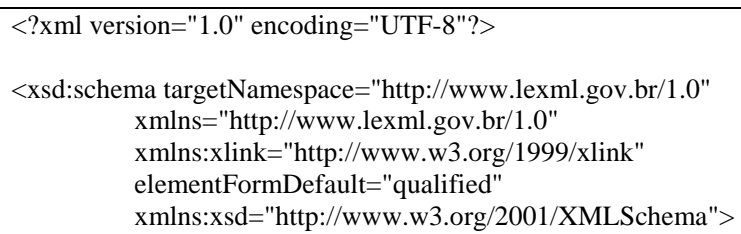

<xsd:import namespace="http://www.w3.org/1999/xlink" schemaLocation="Xlink.xsd" />

<xsd:import namespace="http://www.w3.org/XML/1998/namespace" schemaLocation="http://www.w3.org/2001/xml.xsd"/>

$<$ xsd:include schemaLocation="lexml-base.xsd"/>

$<$ xsd:simpleType name="idArtigo" $>$

$<$ xsd:annotation>

$<$ xsd:documentation>

A regras abaixo tratam dos IDs de dispositivos no nível de artigo para baixo. $</$ xsd:documentation $>$

$</$ xsd:annotation $>$

$<$ xsd:restriction base="xsd:ID"> $<$ xsd:pattern

value="(art $\backslash d+((-[0-9]\{1,3\}) ?)\{0,3\}\left(\_\right.$cpt|_(par|dpg) $) d+((-[0-$

$9]\{1,3\})$ ?) $\left.\{0,3\}) \_a s p \backslash d+\_\right)$?art $\backslash d+((-[0-9]\{1,3\})$ ?) $\{0,3\} "></$ xsd:pattern $>$ $<$ xsd:pattern $\quad$ value="(art $\backslash d+((-[0-9]\{1,3\}))\{0,3\}\left(\_c p t \mid(p a r \mid d p g) \backslash d+((-[0-\right.$

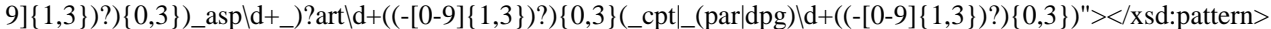
$<$ xsd:pattern value="(artld+((-[0-9]\{1,3\})?)\{0,3\}(_cpt|_(par|dpg) $\backslash \mathrm{d}+((-[0-$ $9]\{1,3\})$ ?) $\{0,3\}) \_$asp $\left.\backslash d+\_\right)$?art $\backslash d+((-[0-9]\{1,3\})$ ? $)\{0,3\}\left(\_c p t \mid(\right.$ par $\mid \mathrm{dpg}) \backslash \mathrm{d}+((-[0-9]\{1,3\})$ ?) $\{0,3\}) \_(\mathrm{inc} \mid \mathrm{dpg}) \backslash \mathrm{d}+((-[0-$ 9] $\{1,3\})$ ?) $\{0,3\} "></$ xsd:pattern $>$

<xsd:pattern $\quad$ value="(art $\quad$ d $+((-[0-9]\{1,3\}) ?)\{0,3\}\left(\_c p t \mid \_(p a r \mid d p g) \backslash d+((-[0-\right.$ 9] $\{1,3\})$ ?) $\{0,3\}) \_$asp $\left.\backslash d+\_\right)$?art $\backslash d+((-[0-9]\{1,3\})$ ? $)\{0,3\}($ cpt|_(par|dpg $) \backslash d+((-[0-9]\{1,3\})$ ?) $\{0,3\}) \_(\text {inc } \mid \operatorname{dpg}) \backslash d+((-[0-$ 9] $\{1,3\})$ ?) $\{0,3\} \_(\text {ali } \mid \mathrm{dpg}) \backslash \mathrm{d}+((-[0-9]\{1,3\})$ ? $)\{0,3\} "></$ xsd:pattern $>$

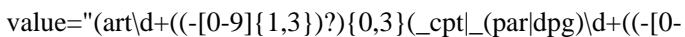

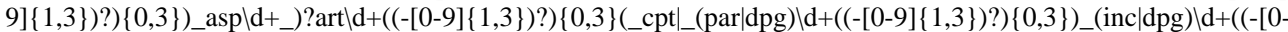
$9]\{1,3\})$ ?) $\{0,3\} \_(\text {ali } \mid \mathrm{dpg}) \backslash \mathrm{d}+((-[0-9]\{1,3\})$ ? $)\{0,3\} \_(\text {ite } \mid \mathrm{dpg}) \backslash \mathrm{d}+((-[0-9]\{1,3\})$ ? $)\{0,3\} "></$ xsd:pattern $>$

$</$ xsd:restriction>

$</$ xsd:simpleType $>$

$<$ xsd:simpleType name="idAgregador" $>$ $<$ xsd:annotation> $<$ xsd:documentation>

A regras abaixo tratam dos IDs de dispositivos no nível de artigo para cima. $</$ xsd:documentation $>$

$</$ xsd:annotation $>$

$<$ xsd:restriction base="xsd:ID"> $<$ xsd:pattern value $="\left(\operatorname{art} \backslash d+((-[0-9]\{1,3\}) ?)\{0,3\}\left(\_c p t \mid \_(p a r \mid d p g) \backslash d+((-[0-\right.\right.$ 9]\{1,3\})?)\{0,3\})_asp $\left.\backslash d+\_\right)$(prt|agh) $\backslash d+((-[0-9]\{1,3\})$ ? $)\{0,3\} "></$ xsd:pattern $>$ $<$ xsd:pattern

value $="\left(\operatorname{art} \backslash d+((-[0-9]\{1,3\}) ?)\{0,3\}\left(\_c p t \mid \_(p a r \mid d p g) \backslash d+((-[0-\right.\right.$

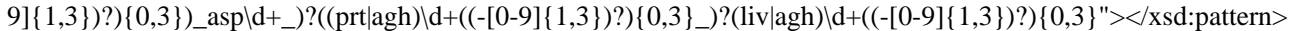
$<$ xsd:pattern value="(art $\quad$ d $+((-[0-9]\{1,3\})$ ?) $\{0,3\}($ cpt|_(par|dpg) $\backslash \mathrm{d}+((-[0-$ $\left.9]\{1,3\}) ?)\{0,3\}) \_a s p \backslash d+\_\right) ?\left((\right.$ prt $\mid$ agh $\left.\left.) \backslash d+((-[0-9]\{1,3\}) ?)\{0,3\}_{-}\right) ?(\operatorname{liv} \mid a g h) \backslash d+((-[0-9]\{1,3\}) ?)\{0,3\} \_\right) ?(\operatorname{tit} \mid \operatorname{agh}) \backslash d+((-[0-$ 9] $\{1,3\})$ ?) $\{0,3\} "></$ xsd:pattern $>$ <xsd:pattern $\quad$ value="(art $\quad$ d + $((-[0-9]\{1,3\}) ?)\{0,3\}\left(\_c p t \mid(p a r \mid d p g) \backslash d+((-[0-\right.$ $\left.9]\{1,3\}) ?)\{0,3\}) \_a s p \backslash d+\_\right) ?\left(\left(\left((p r t \mid a g h) \backslash d+((-[0-9]\{1,3\}) ?)\{0,3\}_{-}\right) ?(\operatorname{liv} \mid\right.\right.$ agh $\left.) \backslash d+((-[0-9]\{1,3\}) ?)\{0,3\} \_\right) ?(\operatorname{tit} \mid \operatorname{agh}) \backslash d+((-[0-$ 9] $\{1,3\})$ ?) $\left.\{0,3\}_{-}\right)$?(cap|agh) $\backslash d+((-[0-9]\{1,3\})$ ? $)\{0,3\} "></$ xsd:pattern $>$ $<$ xsd:pattern value="(art $\backslash d+((-[0-9]\{1,3\})$ ?) $\{0,3\}($ cpt $\mid$ (par $\mid \mathrm{dpg}) \backslash \mathrm{d}+((-[0-$ $9]\{1,3\})$ ? $\left.)\{0,3\}) \_a s p \backslash d+\_\right) ?\left(\left(\left((\right.\right.\right.$ prt $\mid$ agh $) \backslash d+((-[0-9]\{1,3\})$ ? $\left.)\{0,3\}_{-}\right)$? $\left.(\operatorname{liv} \mid \operatorname{agh}) \backslash d+((-[0-9]\{1,3\}) ?)\{0,3\} \_\right) ?($ tit $\mid$ agh $) \backslash d+((-[0-$ $9]\{1,3\})$ ?) $\left.\{0,3\}_{\_}\right)$?(cap|agh $\backslash \mathrm{d}+((-[0-9]\{1,3\})$ ? $\left.)\{0,3\}_{-}\right)$?(sec|agh $) \backslash \mathrm{d}+((-[0-9]\{1,3\})$ ? $)\{0,3\} "></$ xsd:pattern $>$ $<$ xsd:pattern value="(artld+((-[0-9]\{1,3\})?)\{0,3\}(_cpt|_(par|dpg) $\backslash \mathrm{d}+((-[0-$ $\left.9]\{1,3\}) ?)\{0,3\}) \_a s p \backslash d+\_\right) ?\left(\left(\left((\right.\right.\right.$ prt $\mid$ agh $) \backslash d+((-[0-9]\{1,3\})$ ? $\left.)\{0,3\}_{-}\right)$? $\left.(\operatorname{liv} \mid \operatorname{agh}) \backslash d+((-[0-9]\{1,3\}) ?)\{0,3\} \_\right) ?($ tit $\mid$ agh $) \backslash d+((-[0-$ $9]\{1,3\})$ ? $\left.)\{0,3\} \_\right)$?(cap|agh $\left.) \backslash d+((-[0-9]\{1,3\}) ?)\{0,3\}_{-}\right)$?(sec|agh $) \backslash d+((-[0-9]\{1,3\}) ?)\{0,3\} \_(\text {sub } \mid \text { agh }) \backslash d+((-[0-$ $9]\{1,3\})$ ?) $\{0,3\} "></$ xsd:pattern $>$

$<$ xsd:simpleType $>$ $</$ xsd:restriction $>$

$<$ xsd:annotation>

$<$ xsd:documentation $>$

Elements for hierarchical documents 


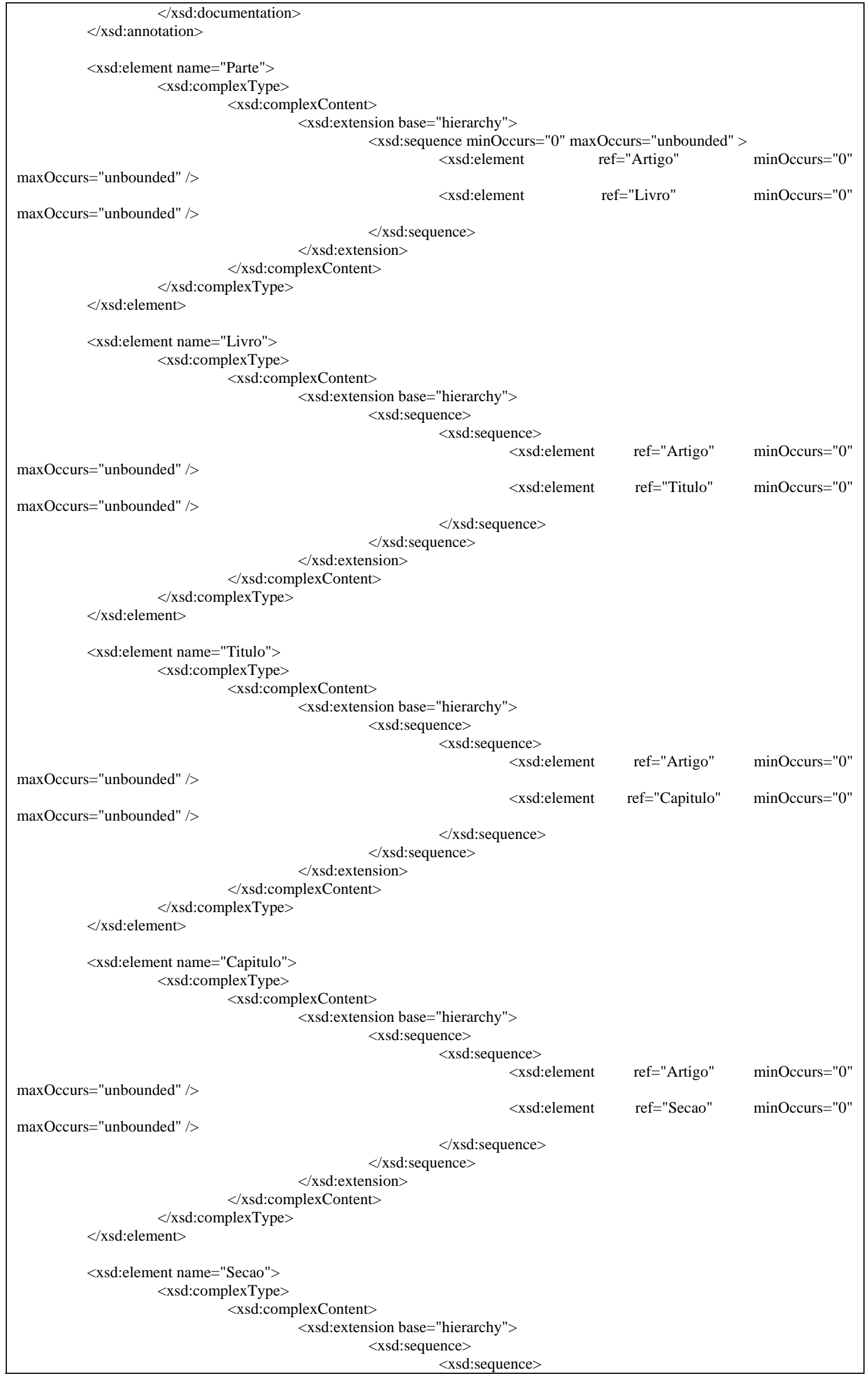




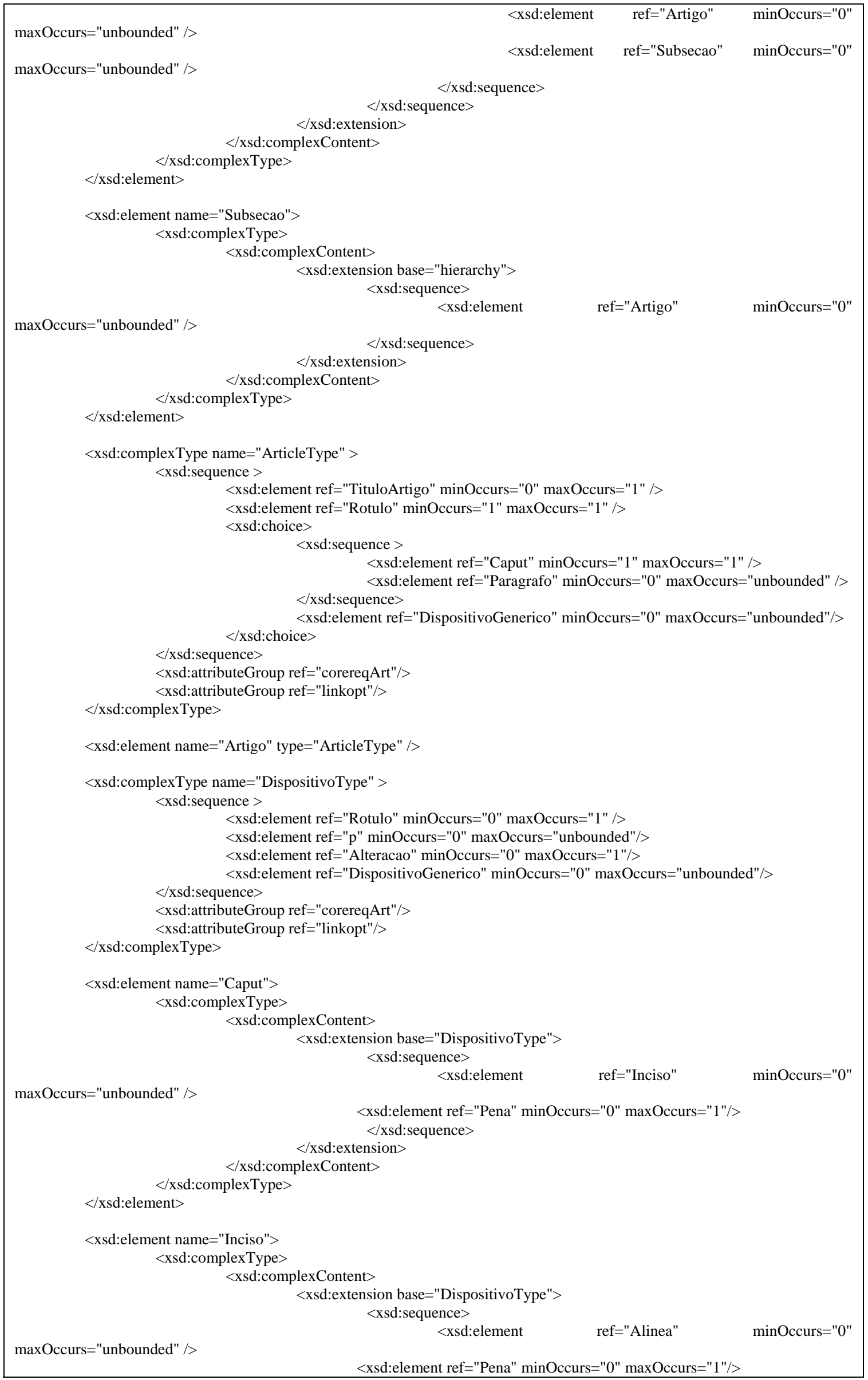




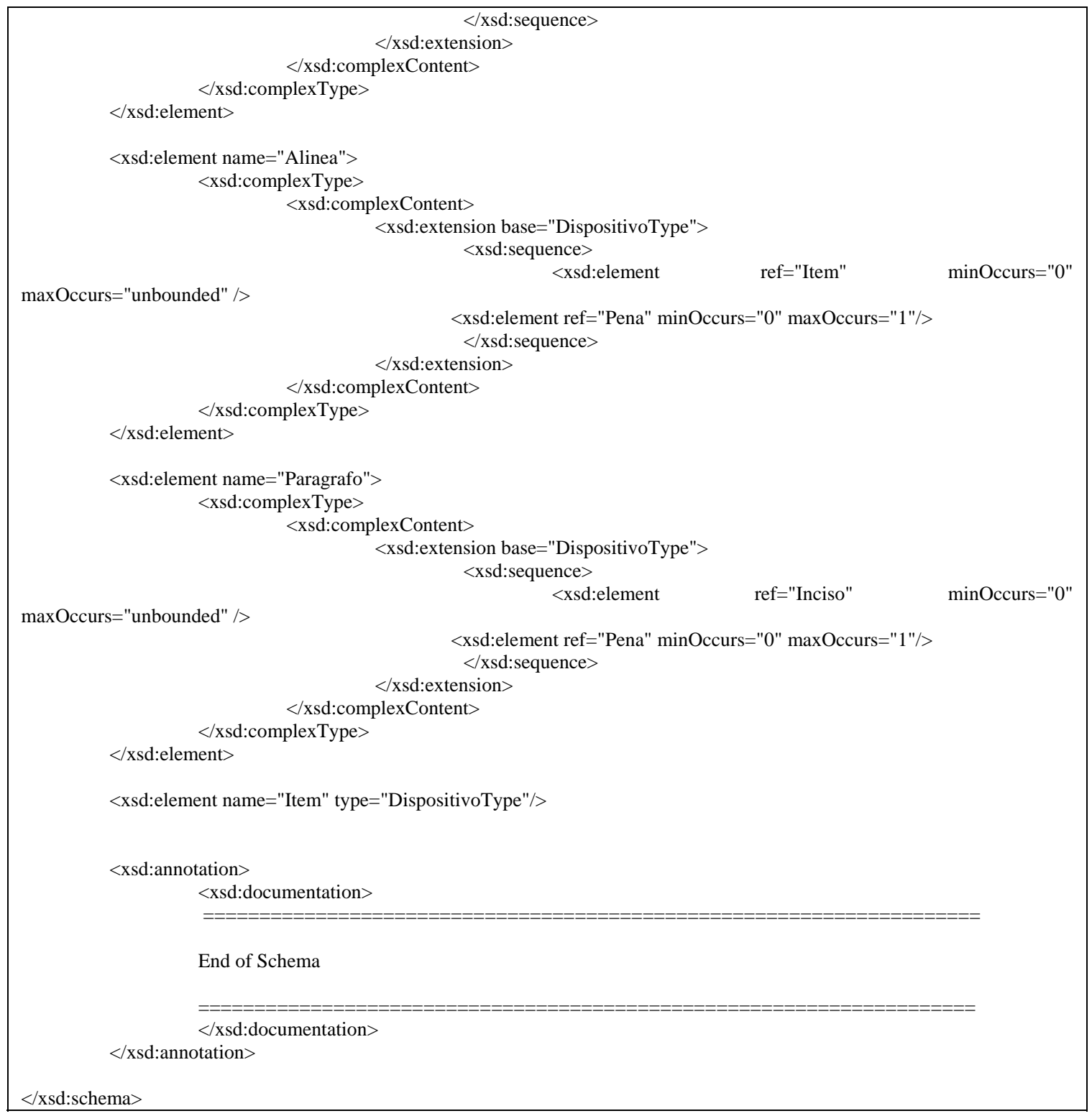




\section{Anexo 3 - Esquema lexml-flexivel.xsd}

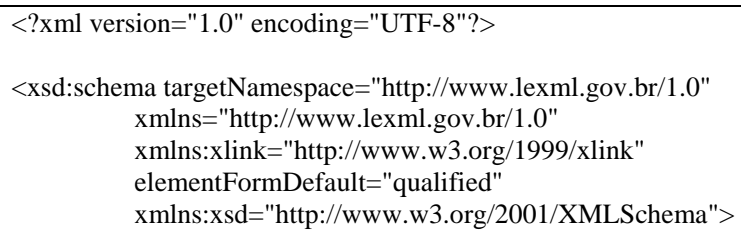

<xsd:import namespace="http://www.w3.org/1999/xlink" schemaLocation="Xlink.xsd" />

<xsd:import namespace="http://www.w3.org/XML/1998/namespace" schemaLocation="http://www.w3.org/2001/xml.xsd"/>

$<$ xsd:include schemaLocation="lexml-base.xsd"/>

$<$ xsd:simpleType name="idArtigo" $>$

$<$ xsd:annotation>

$<$ xsd:documentation>

A regras abaixo tratam dos IDs de dispositivos no nível de artigo para baixo.

TBD: flexibilizar regras de validação dos ids $</$ xsd:documentation $>$

$</$ xsd:annotation $>$

$<$ xsd:restriction base="xsd:ID"> $<$ xsd:pattern $\quad$ value="(art $\backslash d+((-[0-9]\{1,3\})$ ?) $\{0,3\}($ c cpt|_(par|dpg) $\backslash \mathrm{d}+((-[0-$ 9] $\{1,3\})$ ?) $\{0,3\}) \_$asp $\left.\backslash d+\_\right)$?art $\backslash d+((-[0-9]\{1,3\})$ ? $)\{0,3\} "></$ xsd:pattern $>$ $<$ xsd:pattern $\quad$ value="(artld+((-[0-9]\{1,3\})?)\{0,3\}( cpt| (par|dpg) $\backslash \mathrm{d}+((-[0-$

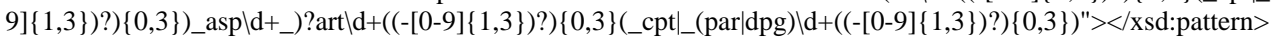
$<$ xsd:pattern value $="(\operatorname{artld}+((-[0-9]\{1,3\})$ ? $)\{0,3\}($ cpt|_(p

9] $\{1,3\})$ ?) $\{0,3\})$ asp $\backslash \mathrm{d}+$ + $)$ ?art $\backslash \mathrm{d}+((-[0-9]\{1,3\})$ ?) $\{0,3\}$ ( cpt|_(par|dpg) $\backslash \mathrm{d}+((-[0-9]\{1,3\})$ ?) $\{0,3\})($ inc $\mid \mathrm{dpg}) \backslash \mathrm{d}+((-[0-$

$9]\{1,3\})$ ?) $\{0,3\} "></$ xsd:pattern $>$

$<$ xsd:pattern $\quad$ value="(art $\backslash d+((-[0-9]\{1,3\})$ ?) $\{0,3\}($ cpt|_(par|dpg) $\backslash \mathrm{d}+((-[0-$ 9] $\{1,3\})$ ?) $\{0,3\}) \_$asp $\left.\backslash d+\_\right)$?art $\backslash d+((-[0-9]\{1,3\})$ ? $)\{0,3\}\left(\_c p t \mid(\right.$ par $\mid \mathrm{dpg}) \backslash d+((-[0-9]\{1,3\})$ ?) $\{0,3\}) \_(\text {inc } \mid \mathrm{dpg}) \backslash \mathrm{d}+((-[0-$

$9]\{1,3\})$ ?) $\{0,3\} \_(\text {ali } \mid \mathrm{dpg}) \backslash \mathrm{d}+((-[0-9]\{1,3\}) ?)\{0,3\} "></$ xsd:pattern $>$

$<$ xsd:pattern $\quad$ value="(art $\backslash d+\left((-[0-9]\{1,3\})\right.$ ?) $\{0,3\}\left(\_c p t \mid \_(p a r \mid d p g) \backslash d+((-[0-\right.$

$9]\{1,3\})$ ?) $\{0,3\}) \_$asp $\left.\backslash d+\_\right)$?art $\backslash d+((-[0-9]\{1,3\}) ?)\{0,3\}\left(\_c p t \mid(p a r \mid d p g) \backslash d+((-[0-9]\{1,3\})\right.$ ? $\left.)\{0,3\}\right) \_(\text {inc } \mid \mathrm{dpg}) \backslash \mathrm{d}+((-[0-$

9] $\{1,3\})$ ?) $\{0,3\} \_(\text {ali } \mid \mathrm{dpg}) \backslash \mathrm{d}+((-[0-9]\{1,3\})$ ?) $\{0,3\}$ _(ite|dpg $) \backslash \mathrm{d}+((-[0-9]\{1,3\})$ ?) $\{0,3\} "></$ xsd:pattern $>$

$</$ xsd:restriction>

$</$ xsd:simpleType $>$

<xsd:simpleType name="idAgregador"> $<$ xsd:annotation $>$

$<$ xsd:documentation $>$

A regras abaixo tratam dos IDs de dispositivos no nível de artigo para cima.

TBD: flexibilizar regras de validação dos ids $</$ xsd:documentation $>$

$</$ xsd:annotation $>$

$<$ xsd:restriction base="xsd:ID"> $<$ xsd:pattern value $="\left(\operatorname{art} \backslash d+((-[0-9]\{1,3\}) ?)\{0,3\}\left(\_c p t \mid \_(p a r \mid d p g) \backslash d+((-[0-\right.\right.$ 9] $\{1,3\})$ ?) $\{0,3\})$ asp $\backslash d+)$ ? (prt|agh) $\backslash d+((-[0-9]\{1,3\})$ ?) $\{0,3\} "></$ xsd:pattern $>$ $<$ xsd:pattern value $="\left(\operatorname{art} \backslash d+((-[0-9]\{1,3\}) ?)\{0,3\}\left(\_c p t \mid \_(p a r \mid d p g) \backslash d+((-[0-\right.\right.$ 9] $\{1,3\})$ ?) $\left.\{0,3\}) \_a s p \backslash d+\_\right)$?(prt|agh $) \backslash d+((-[0-9]\{1,3\})$ ? $\left.)\{0,3\} \_\right)$?(liv|agh $) \backslash d+((-[0-9]\{1,3\})$ ?) $\{0,3\} "></$ xsd:pattern $>$ <xsd:pattern value="(art $\quad$ dd+((-[0-9] $\{1,3\})$ ?) $\{0,3\}($ cpt|_(par|dpg) $\backslash \mathrm{d}+((-[0-$ $\left.9]\{1,3\}) ?)\{0,3\}) \_a s p \backslash d+\_\right) ?\left(\left((p r t \mid a g h) \backslash d+((-[0-9]\{1,3\})\right.\right.$ ? $\left.)\{0,3\} \_\right) ?(\operatorname{liv} \mid$ agh $\left.) \backslash d+((-[0-9]\{1,3\}) ?)\{0,3\} \_\right) ?($ tit $\mid$ agh $) \backslash d+((-[0-$ $9]\{1,3\})$ ?) $\{0,3\} "></$ xsd:pattern $>$ <xsd:pattern $\quad$ value="(art $\backslash d+((-[0-9]\{1,3\}) ?)\{0,3\}\left(\_c p t \mid \_(p a r \mid d p g) \backslash d+((-[0-\right.$

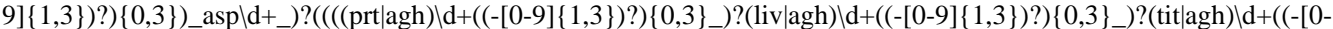
$9]\{1,3\})$ ? $\left.)\{0,3\}_{-}\right)$?(cap|agh)\d+((-[0-9]\{1,3\})?)\{0,3\}"></xsd:pattern> $<$ xsd:pattern

value="(art $\mathbf{d}+((-[0-9]\{1,3\}) ?)\{0,3\}\left(\_c p t \mid \_(p a r \mid d p g) \backslash d+((-[0-\right.$

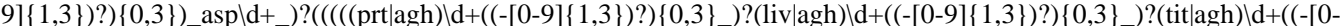
$9]\{1,3\})$ ? $\left.)\{0,3\}_{-}\right)$?(caplagh) $\backslash d+((-[0-9]\{1,3\})$ ? $\left.)\{0,3\}_{-}\right)$?(sec|agh $) \backslash d+((-[0-9]\{1,3\})$ ? $)\{0,3\}$ " ></xsd:pattern> $<$ xsd:pattern value $="(\operatorname{art} \backslash d+((-[0-9]\{1,3\}) ?)\{0,3\}($ cpt $\mid($ par|dpg $) \backslash d+((-[0-$

$9]\{1,3\})$ ? $\left.\{\{0,3\}) \_a s p \backslash d+\_\right) ?\left(\left(\left((\right.\right.\right.$ prt $\mid$ agh $\left.\left.) \backslash d+((-[0-9]\{1,3\}) ?)\{0,3\} \_\right) ?(\operatorname{liv} \mid \operatorname{agh}) \backslash d+((-[0-9]\{1,3\}) ?)\{0,3\} \_\right) ?(\operatorname{tit} \mid a g h) \backslash d+((-[0-$ $9]\{1,3\})$ ?) $\left.\{0,3\}_{-}\right)$?(cap $\mid$agh $) \backslash \mathrm{d}+((-[0-9]\{1,3\})$ ? $\left.)\{0,3\}_{-}\right)$?(sec|agh $) \backslash \mathrm{d}+((-[0-9]\{1,3\}) ?)\{0,3\} \_(\operatorname{sub} \mid \mathrm{agh}) \backslash \mathrm{d}+((-[0-$ 9] $\{1,3\})$ ?) $\{0,3\} "></$ xsd:pattern $>$ $</$ xsd:restriction $>$ $</$ xsd:simpleType $>$

$<$ xsd:annotation>

$<$ xsd:documentation $>$ 


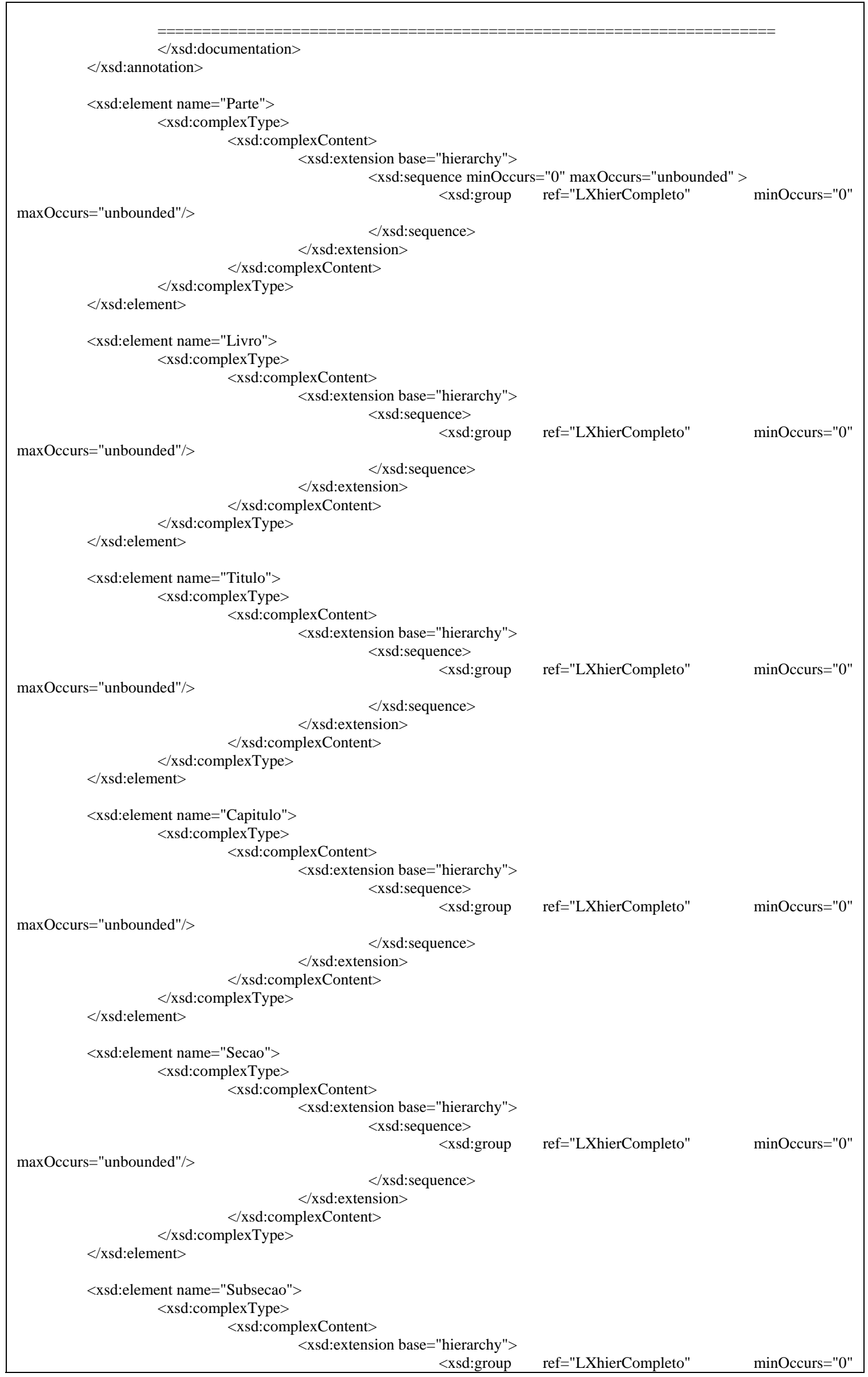




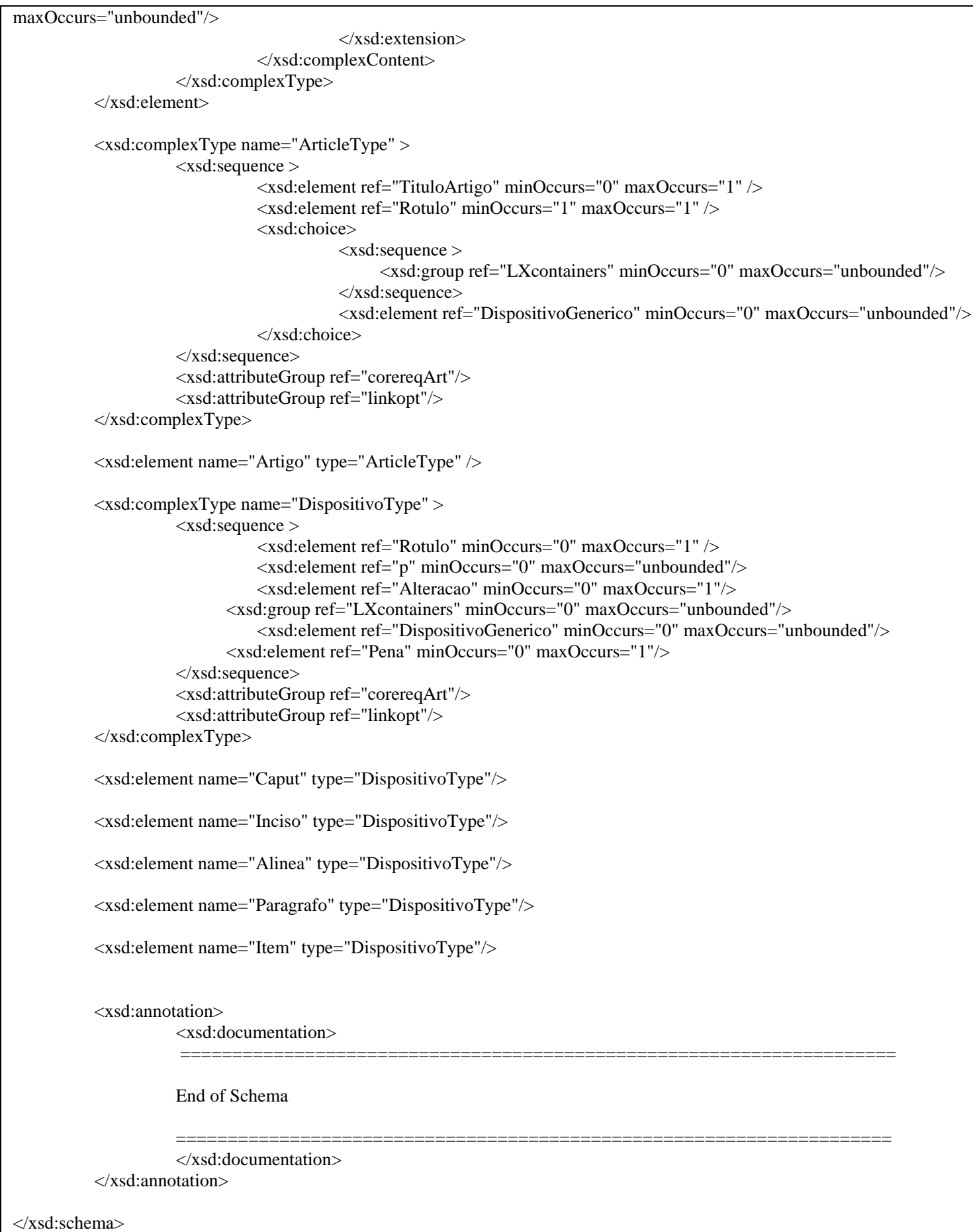

